Research paper

\title{
Comparative analysis of two sister Erythrophleum species (Leguminosae) reveal contrasting transcriptome-wide responses to early drought stress
}

\author{
Mohamed Neji $^{\mathrm{a}, \mathrm{g}, \mathrm{h}, *}$, Anais Gorel $^{\mathrm{b}}$, Dario I. Ojeda ${ }^{\mathrm{a}, \mathrm{e}, \mathrm{f}}$, Jérôme Duminil ${ }^{\mathrm{a}, \mathrm{c}}$, Chedly Kastally ${ }^{\mathrm{a}}$, \\ Kathy Steppe ${ }^{\mathrm{d}}$, Adeline Fayolle ${ }^{\mathrm{b}}$, Olivier J. Hardy ${ }^{\mathrm{a}}$ \\ ${ }^{a}$ Unit of Evolutionary Biology \& Ecology, Faculté des Sciences, Université Libre de Bruxelles, Av. F.D. Roosevelt, 50, CP 160/12, B-1050 Brussels, Belgium \\ ${ }^{\mathrm{b}}$ Department Biosystem Engineering (BIOSE), Gembloux Agro-Bio Tech, University of Liege, Belgium \\ ${ }^{\mathrm{C}}$ UMR-DIADE, Institut de Recherche pour le Développement, Univ. Montpellier, Montpellier, France \\ ${ }^{\mathrm{d}}$ Laboratory of Plant Ecology, Faculty of Bioscience Engineering, Ghent University, Coupure links 653, B-9000 Ghent, Belgium \\ ${ }^{\mathrm{e}}$ Unit of Ecology and Genetics, Department of Biology, Oulu University, Finland \\ ${ }^{\mathrm{f}}$ Norwegian Institute of Bioeconomy Research, Ås, Norway \\ ${ }^{g}$ Department of Life Sciences, Faculty of Sciences of Gabès, University of Gabès, Tunisia \\ ${ }^{\mathrm{h}}$ Laboratory of Extremophile Plants, Centre of Biotechnology of Borj Cedria, Hammam-Lif, Tunisia
}

\section{A R T I C L E I N F O}

\section{Keywords:}

African rainforests

Erythrophleum

RNA-seq

Transcriptomic

Differentially expressed genes

Drought tolerance

\begin{abstract}
A B S T R A C T
With the ongoing climate change, African rainforests are expected to experience severe drought events in the future. In Africa, the tropical genus Erythrophleum (Fabaceae) includes two forest sister timber tree species displaying contrasting geographical distributions. Erythrophleum ivorense is adapted to wet evergreen GuineoCongolian forests, whereas E. suaveolens occurs in a wider range of climates, being found in moist dense forests but also in gallery forests under a relatively drier climate. This geographical distribution pattern suggests that the two species might cope differently to drought at the genomic level. Yet, the genetic basis of tolerance response to drought stress in both species is still uncharacterized. To bridge this gap, we performed an RNA-seq approach on seedlings from each species to monitor their transcriptional responses at different levels of drought stress (0, 2 and 6 weeks after stopping watering seedlings).

Monitoring of wilting symptoms revealed that E. suaveolens displayed an earlier phenotypic response to drought stress than E. ivorense. At the transcriptomic level, results revealed 2020 (1204 down-regulated/816 upregulated) and 1495 differentially expressed genes (DEGs) in response to drought stress from a total of 67,432 and 66,605 contigs assembled in E. ivorense and E. suaveolens, respectively. After identifying 30,374 orthologs between species, we found that only 7 of them were DEGs shared between species, while 587 and 458 were differentially expressed only in E. ivorense or E. suaveolens, respectively. GO and KEGG enrichment analysis revealed that the two species differ in terms of significantly regulated pathways as well as the number and expression profile of DEGs (Up/Down) associated with each pathway in the two stress stages. Our results suggested that the two studied species react differently to drought. E. suaveolens seems displaying a prompt response to drought at its early stage strengthened by the down-regulation of many DEGs encoding for signaling and metabolism-related pathways. A considerable up-regulation of these pathways was also found in E. ivorense at the late stage of drought, suggesting this species may be a late responder. Overall, our data may serve as basis for further understanding the genetic control of drought tolerance in tropical trees and favor the selection of crucial genes for genetically enhancing drought resistance.
\end{abstract}

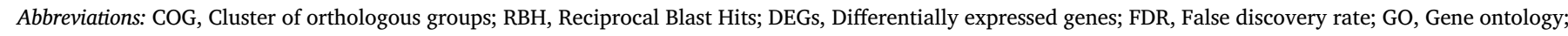

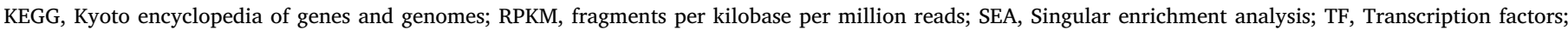
TPM, Transcript per million; NMDS, Non-metric Multidimensional Scaling

* Corresponding author at: Unit of Evolutionary Biology \& Ecology, Faculté des Sciences, Université Libre de Bruxelles, Av. F.D. Roosevelt, 50, CP 160/12, B-1050 Brussels, Belgium.

E-mail address: mohamed.neji@ulb.ac.be (M. Neji). 


\section{Introduction}

Drought has been long recognized as one of the most important environmental factors driving the geographical distribution of plant species. It poses tremendous threat to sustainable agriculture and forestry worldwide as it severely impairs the survival of plants, mainly in water-limited ecosystems (Liu et al., 2017; Yang et al., 2018). Tropical ecosystems support around half of all terrestrial plant and animal species, including $96 \%$ of tree species, and represent $34 \%$ of gross primary terrestrial productivity (Corlett, 2016). Drought events frequency and water scarcity are predicted to increase progressively as an outcome of global climatic changes (Wuebbles et al., 2014; Sprenger et al., 2016), with profound impacts on the vegetation of the Amazonian, Asian and African tropical regions (Solomon et al., 2007; Buytaert et al., 2011; Kirtman et al., 2013; Bonal et al., 2016). Nowadays, African rainforests are subject to deforestation and habitat degradation (Mayaux et al., 2004; Duveiller et al., 2008), which make them highly susceptible to droughts resulting from the expected climatic changes.

The tropical genus Erythrophleum includes two large sister tree species geographically widespread in the tropical African rainforest and characterized by their high economic and socio-cultural value: $E$. suaveolens and E. ivorense (Fabaceae-Caesalpinioideae). Although they are morphologically very similar to each other, these species display a parapatric distribution and grow in different climatic environments, representing a wide range of biotic and abiotic conditions that may be associated with adaptive natural genetic variation (Duminil et al., 2010). Erythrophleum ivorense is restricted to the wet and evergreen Guineo-Congolian forests ( $>2000 \mathrm{~mm}$ rainfall) bordering the gulf of Guinea, from Guinea to Gabon. In contrast, E. suaveolens has a wide geographical distribution extending from Senegal east to Sudan and Kenya, and south to Mozambique and Zimbabwe, and is adapted to the more seasonal climate of semi-deciduous Guineo-Congolian forests ( $>1600 \mathrm{~mm}$ rainfall) and the drier climate of forest-savanna mosaic landscapes (1100-1600 mm rainfall) (Vivien and Faure, 1985; Akoègninou et al., 2006; Duminil et al., 2013). The respective geographical distributions of the two species suggest that they probably respond to environmental stresses differently at the genomic level. We therefore hypothesized that these sister species may use divergent regulatory and metabolic pathways during their interaction with different abiotic constraints.

Due to their contrasting ecological features, these species are promising evolutionary models of rainforest species. Recent investigations, mainly focusing on the patterns of genetic variation and demographic changes, have pointed out a substantial spatial genetic structure in these species, associated to rainfall gradients and past climatic changes (Duminil et al., 2010; Duminil et al., 2013; Duminil et al., 2015). However, the genetic bases and molecular mechanisms underlying their response to environmental variation have not been yet investigated, while sister species adapted to contrasted environments offer the possibility to compare the expression of very similar genomes showing different adaptations. Here we assume that the wider geographical distribution of E. suaveolens, compared to E. ivorense, might be attributed to its better adaptation to severe environmental constraints, including drought.

Deciphering the underlying molecular mechanism of plant responses under drought stress remains a major challenge in biology. Drought tolerance involving the interplay of a vast array of mechanisms, its genetic control is difficult to determine without detailed genetic and sequence information (Liang et al., 2017; Tricker et al., 2018). In general, drought resistance in plants could be related to various molecular mechanisms controlled at hormonal and transcriptomic level (Yildirim and Kaya, 2017). According to Yates et al. (2014), the typical response to drought is to reduce the water loss by closing stomata and diminishing photosynthesis. At the molecular level, plants react to drought by changing the expression profiles of a large set of genes encoding for several pathways and transcription factors (TFs) with up- as well as down-regulation (Gao et al., 2015; Jia et al., 2016). The response starts by a step of stress perception that triggers common pathways ('plant hormone signal transduction', 'Calcium signaling pathway' and 'MAPK signaling pathway'), and many TFs (ATHB7, NAC and WRKY) involved in signal transduction. Then, a modification in expression profiles occurs in a cascade of genes involved in several pathways related to carbohydrate metabolism, oxidative phosphorilation, glycolysis, photosynthesis, transcription, nucleotide and antioxidants metabolisms in order to adjust the reduced water.

A deep transcriptomic analysis, via massively parallel cDNA sequencing technology, or RNA sequencing (RNA-seq), could provide detailed information about gene expression at the mRNA level. Nowadays, this approach is widely used for quantitative transcriptome profiling, accurate quantification of gene expression and detecting differentially expressed genes in order to unravel a diversity of stress responses at a transcriptome-wide level. RNA-seq is a cost and timeeffective approach, and it has been recently used to analyze global gene expression in plant responses to various abiotic stresses such as drought (Huang et al., 2015; Fracasso et al., 2016; de Freitas Guedes et al., 2018), cold and salt for model and non-model plant species.

In the present study, using an RNA-seq approach, our major interest was to characterize the leaf transcriptomes of $E$. suaveolens and $E$. ivorense seedlings under drought stress in order to: 1) characterize the immediate gene expression response to mild short-term drought stress in the two species at the genome wide level, 2) identify the strategy employed to cope with severe drought-stress exposure and the main metabolic pathways involved, and 3) determine the differences in drought response between the two species.

\section{Material and methods}

\subsection{Plant material and drought stress experiment}

Seeds of E. ivorense and E. suaveolens used for the experiment were collected from mother trees originating from two different localities in Cameroon: Korup ( $\left.5^{\circ} 03^{\prime} 55^{\prime \prime} \mathrm{N} ; 8^{\circ} 51^{\prime} 28^{\prime \prime} \mathrm{E}\right)$ and Mindourou $\left(3^{\circ} 23^{\prime}\right.$ $03^{\prime \prime} \mathrm{N} ; 14^{\circ} 30^{\prime} 20^{\prime \prime} \mathrm{E}$ ), respectively. Collected seeds were first grown in a greenhouse at Gembloux Agro-Bio Tech, Belgium for ten months. In October 2016, seedlings were moved to a controlled glasshouse set to $25^{\circ} \mathrm{C}$ for 12 -h days and $22^{\circ} \mathrm{C}$ for 12 -h nights at the Laboratory of Plant Ecology, Faculty of Bioscience Engineering, Ghent University, Belgium, to conduct ecophysiological measurements on 40 seedlings. Here, we describe only the components of this experiment relevant for our gene expression study. Plants were well irrigated for 30 days prior to the drought treatment. After acclimation, seedlings were equally divided into two groups: well-watered plants (the control plants) and waterdeprived plants (the drought-stressed plants) and arranged in a randomized block design. The drought stress experiment started on November 1st, 2016 and continued for 10 weeks. For gene expression analysis, leaf samples were collected from two similarly vigorous seedlings from each species at three different times during the experiment: before drought treatment (T0), after two weeks (T1) and six weeks (T2) of drought stress. Leaves were harvested and immediately frozen in liquid nitrogen and stored at $-80^{\circ} \mathrm{C}$ for RNA extraction. Symptoms of stress were monitored weekly by visual inspection of the signs of wilting in all seedlings according to the $0-5$ scale of Tyree et al. (2002). The soil water content was also recorded weekly using time domain reflectometry (TDR) as previously reported (Moret-Fernández et al., 2012). Stages T1 and T2 were considered as the mild droughtstress and severe drought-stress, respectively (see results).

\subsection{RNA extraction, cDNA library preparation and sequencing}

Total RNA was extracted from frozen tissues using Plant RNA reagent (Invitrogen, Carlsbad, USA) according to the manufacturer's instructions and treated with RNase-free DNase to remove the residual 
genomic DNA. The integrity and the concentration of the extracted RNA were checked by denaturing agarose gel electrophoresis (1\%) and quantified by a Nanodrop spectrophotometer and Qubit (Invitrogen, Ltd., UK). Equal amount of total RNA $(20 \mu \mathrm{g})$ was then used for cDNA library construction using NEXTflex ${ }^{\mathrm{TM}}$ Rapid Directional RNA-Seq Kit (Bioo Scientific, USA) according to manufacturer's instructions. Briefly, the mRNA was purified from the total RNA using Oligo (dT) magnetic beads. Fragmentation buffer was then added to break mRNA into short pieces. The cleaved mRNA fragments were reverse transcribed into first-strand cDNA, then second-strand cDNA, using random primers and transcriptase. The double-stranded cDNA fragments were purified by AMPure XP beads. After adenylation of 3' ends of DNA fragments, the resultant double-stranded cDNA were end-repaired and ligated to specific sequencing adaptors. cDNA fragments were then purified using AMPure XP beads and enriched by PCR to get the final cDNA library for transcriptome sequencing. The concentration and the quality of libraries were checked using Qubit fluorometer (Life Technologies) and QIAxcel (Qiagen). Barcoded libraries were then pooled in equimolar amounts and sequenced in a single lane of an Illumina NextSeq 500 platform at GENOMICS CORE (KULeuven, Belgium) in single-end mode and high output with reads of $75 \mathrm{bp}$ in length.

\subsection{Preprocessing of Illumina reads and de novo transcriptome assembly}

The obtained raw data of each sample were initially cleaned by removing the adapters and filtering out the low-quality sequences $(\mathrm{Q} \leq 5)$ and ambiguous reads sequences (the proportion of $\mathrm{N}>5 \%$ ) using Trimmomatic software (Bolger et al., 2014). Following quality check and normalization of data, for each species the clean data of all samples were merged and de novo assembled using Trinity (Haas et al., 2013) with default parameters and an optimized k-mer length of 25 to generate a reference transcriptome. The program CD-HIT was then used with $98 \%$ of sequence identity and a word size of $10(c=98 \% n=10)$ to eliminate redundant transcripts (Fu et al., 2012). Furthermore, the quality of the assembled transcriptomes was assessed by mapping the reads of individual samples to the assembled transcriptomes using bowtie2 v.2 (-p 10, -q, -no-unal and -k 20) (Langmead and Salzberg, 2012) and the obtained bam files analyzed with samtools (Li et al., 2009). Completeness of the assemblies was determined with BUSCO v.2 using the embryophyta_odb9 data set (Simão et al., 2015). Finally, the assembled contigs on each transcriptome were compared with BLAST + against the Medicago trunculata protein data set (Mt4.0v2) with an $E$ value 1E-20 (Camacho et al., 2009).

\subsection{Identification of orthologs}

The reciprocal-best-BLAST-hits (RBH) has been considered the most robust and effective in identifying the orthologs genes between closely related species (Osada et al., 2008; Elmer et al., 2010; Gao et al., 2018). In our study, we applied this approach to identify orthologs between $E$. ivorense and E. suaveolens. Because the two studied species have diverged recently (Duminil et al., 2015), we directly used nucleotide sequences to run BLAST search instead of peptide sequence in order to ensure accuracy of orthologs identification (An et al., 2016). First, the contigs sets of both species were searched against each other using BLASTN (package of ncbi-blast-2.6.0+) with a bit score $\geq 200$ and an E-value < 1E-50 (Baldo et al., 2011; Lee et al., 2013). Then, to discard putative paralogs, each blast search was parsed to keep hits with at least $98 \%$ sequence identity, and a pair was considered orthologous if the best BLAST hit pointed reciprocally to each other. Moreover, when a gene contained different isoforms following transcriptome assembly by Trinity, we checked that the RBH of each isoform always pointed to the same pairs of Trinity 'genes' in the two species before concluding they were orthologs. This conservative approach might miss some true orthologs but should minimize paralogs.

\subsection{Functional annotation}

All non-redundant transcripts were annotated by searching against the following databases: UniProtKB/Swiss-Prot, Pfam (version 31.0) (Finn et al., 2015), Kyoto Encyclopedia of Genes and Genomes (KEGG: http://www.genome.jp/kegg/) (Kanehisa et al., 2016), and Non-supervised Orthologous Groups (eggNOG) (http://eggnog.embl.de/) (Huerta-Cepas et al., 2015). Transdecoder 2.0.1 (http://transdecoder.sf. net) was first used to identify the protein coding sequences of at least 100 amino acids in length within the generated transcriptomes. The assembled transcripts and the protein coding sequences were then mapped against UniProtKB/Swiss-Prot database with an E-value cutoff of 1E-5 using Blastx and Blastp in the program ncbi-blast-2.6.0+, respectively. The protein coding sequences were also searched for conserved protein domains against the pfam database via HMMER v. 3.1b2 (http://hmmer.org/) (Finn et al., 2011). Using Trinotate software (https://trinotate.github.io/) (Haas et al., 2013), the obtained outputs were loaded into a SQLite database to generate an annotation report in order to perform Gene Ontology (GO) assignments for the annotated transcripts. GO functional classification was then carried out using the Web Gene Ontology Annotation Plot (WEGO: http://wego.genomics. org.cn) software (Ye et al., 2006). For KEGG pathway annotation, the assembled transcripts were mapped against KEGG database using online KASS server (http://www.genome.jp/tools/kaas/) (updated in March 2018) with the single-directional best hit information method (Moriya et al., 2007). Furthermore, the protein coding sequences were analyzed by eggNOG-Mapper in order to identify the Cluster of orthologous groups (COG) (Huerta-Cepas et al., 2017). Finally, the plant transcription factors (TFs) were identified and classified into different families using the iTAK program (Zheng et al., 2016).

\subsection{Gene expression and exploratory analysis}

Gene expression level of the assembled transcripts of each sample was separately estimated using the FPKM (fragments per kilobase per million reads) metric by program RSEM (V 1.2.4), which allows for a quantification of the expression level of each transcript by assessing transcript abundance when mapping RNA-seq reads to the assembled transcriptome (Li and Dewey, 2011). The clean reads of the analyzed samples were realigned to the final transcriptome assembly using Bowtie 2 v. 2 with the aforementioned parameters in order to count the expression level of each transcript (transcript per million, TPM).

To make a first visualization of the differences in gene expression among the different samples of the two species we conducted a Nonmetric Multidimensional Scaling (NMDS) ordination based on a Mahalanobis distance matrix between samples. The distance was computed on a sample/ortholog table containing $\ln (1+$ TPM $)$ values, where TPM was here computed considering only the set of c. $30 \cdot 10^{3}$ orthologs identified (see results).

\subsection{Identification and enrichment analysis of the differentially expressed genes (DEGs)}

For each species, the differentially expressed genes (DEGs) were then screened by comparing gene expression levels between the three stages of stress (T0, T1 and T2) using the edgeR R package (Robinson et al., 2010). Here, we considered all transcripts, irrespective of whether orthologs were identified or not. False discovery rate (FDR) of 0.05 and $\log 2$ (fold change) of 2 were set as the threshold for significantly differential expression.

For the comparative analysis of DEGs between $E$. ivorense and $E$. suaveolens in response to drought, GO enrichment, which is commonly used for elucidating the biological functions of genes (Young et al., 2010), was performed using singular enrichment analysis (SEA). We used the agriGO program (Du et al., 2010) with the default parameters, and the Medicago truncatula (Fabaceae) gene models as background, 


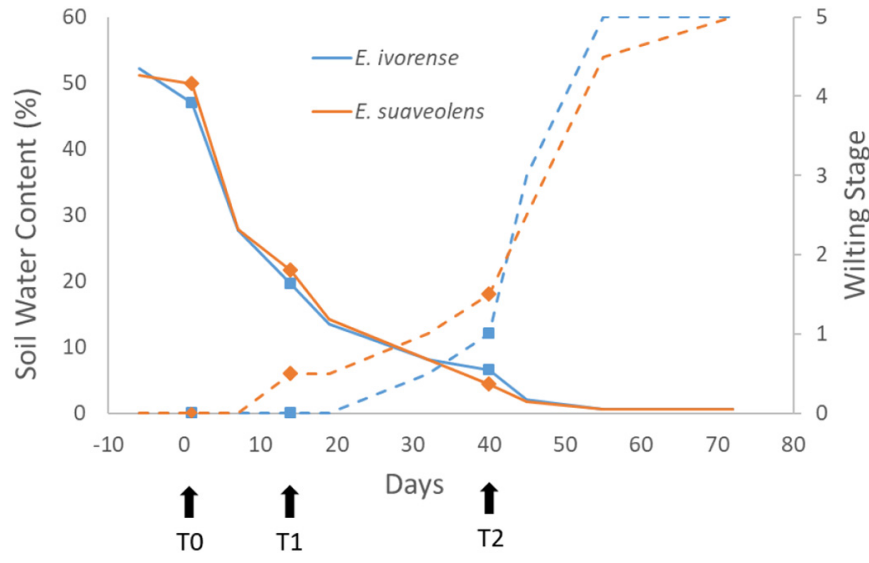

Fig. 1. Effects of drought treatment on soil water content (solid lines) and leaf wilting signs (dashed lines) in E. ivorense (square) and E. suaveolens (diamond). Seedlings were watered until time $=0$. Data are the means of two replicates at each time point. Arrows indicate when leaf samples were collected to analyze their transcriptomes (T0, T1 $=2$ weeks and $\mathrm{T} 2=6$ weeks).

followed by multiple testing with Bonferroni correction (P-value < 0.05). In addition, DEGs involved in specific KEGG molecular pathways were enriched and tested based on the hypergeometric distribution which gives the following P-value: $\mathrm{P}=1-\sum_{i=0}^{x-1} \frac{\left(\begin{array}{c}A \\ i\end{array}\right)\left(\begin{array}{c}B-A \\ y-i\end{array}\right)}{\left(\begin{array}{l}B \\ y\end{array}\right)}$, where $\mathrm{A}$ is the number of annotated genes involved in a specific pathway, B is the number of all genes with KEGG annotation, $x$ is the number of DEGs involved in a specific pathway, and $y$ is the number of DEGs. Only pathways with $\mathrm{P} \leq 0.05$ were considered as significantly enriched. Finally, using the subset of identified orthologs between the two species, we assessed the proportion of orthologs that were differentially expressed in both species.

\section{Results}

\subsection{Phenotypic and physiological response to drought stress}

Results showed that for seedlings of both species the soil water content dropped from c. $50 \%$ in the control samples (T0) to c. $20 \%$ in the 2 weeks-stressed plants and to c. $5 \%$ at 6 weeks of treatment (Fig. 1). We noticed that first signs of wilting (slightly wilted stage) appeared at two weeks in E. suaveolens and at four weeks in E. ivorense (Fig.1). After five weeks, wilting symptoms increased rapidly, especially in E. ivorense, so that the two species displayed the same mean wilting stage at six weeks and $E$. ivorense seedlings were on average more wilted than $E$. suaveolens seedlings after 8 weeks. This pattern was also apparent when considering all seedlings followed for physiological data (results not shown) and may indicate an early drought response in $E$. suaveolens at the morphological and physiological level and thus an earlier initiation of the molecular mechanisms in response to drought stress.

\subsection{Transcriptome sequencing output and assembly}

The sequencing of the 12 pooled libraries generated a total of $181,342,512$ raw single-end reads, covering about $14.78 \mathrm{~Gb}$ of sequencing raw data. After removing adaptors and low quality data, $48,089,328$ and 77,798,254 clean reads were retained for $E$. ivorense and $E$. suaveolens, respectively. De novo assembly of the processed reads yielded 102,420 and 96,600 contigs in E. ivorense and E. suaveolens, respectively. After removing the redundant transcripts, a total of 67,432 and 66,605 contigs were identified for both species, respectively. In both assemblies, the smallest contig length was $402 \mathrm{bp}$, while the
Table 1

Total number of cleaned reads that were mapped to their respective assembled transcriptomes.

\begin{tabular}{llrrr}
\hline Species & Sample & No. of reads & Reads mapped & \multicolumn{1}{c}{$\%$} \\
\hline \multirow{2}{*}{ E. ivorense } & Ivo-C-R1 & $55,495,924$ & $53,913,938$ & 97.15 \\
& Ivo-C-R2 & $1,007,855$ & 987,876 & 98.02 \\
& Ivo-2 weeks-R1 & $10,426,553$ & $10,129,456$ & 97.15 \\
& Ivo-2 weeks-R2 & $1,428,602$ & $1,376,757$ & 96.37 \\
& Ivo-6 weeks-R1 & $1,452,284$ & $1,410,199$ & 97.1 \\
& Ivo-6 weeks-R2 & $7,987,036$ & $7,806,052$ & 97.73 \\
Total & & $77,798,254$ & $75,624,278$ & 97.25 \\
& & $7,134,444$ & $6,863,877$ & 96.21 \\
E. suaveolens & Sua-C-R1 & $12,460,616$ & $12,175,086$ & 97.71 \\
& Sua-C-R2 & $7,568,490$ & $7,352,086$ & 97.14 \\
& Sua-2 weeks-R1 & 769,764 & 748,983 & 96.43 \\
& Sua-2 weeks-R2 & $11,229,990$ & $10,765,568$ & 95.86 \\
& Sua-8 weeks-R2 & $8,926,024$ & $8,507,170$ & 95.31 \\
Total & Sua-8 weeks-R1 & $48,089,328$ & $46,412,770$ & 96.44 \\
\hline
\end{tabular}

largest was 20,242 bp in E. ivorense and $9125 \mathrm{bp}$ in E. suaveolens, and average contig lengths reached $760 \mathrm{bp}$ and $661 \mathrm{bp}$, respectively. The N50 lengths for the transcriptome assembly reached $1005 \mathrm{bp}$ in $E$. ivorense and $1137 \mathrm{bp}$ in E. suaveolens. Additionally, the percentage of G/ $\mathrm{C}$ of the clean reads, usually used as an indicator of closeness between species, was found to be $41 \%$ in both species.

Of the total 1440 BUSCO core genes searched, the assessment of $E$. ivorense transcriptome completeness reached 1027 genes (71\%), consisting of $902(62.6 \%)$ single-copy and 125 (8.7\%) duplicated complete orthologs, with only 130 (9\%) fragmented and 283 (19.7\%) missing orthologs. In E. suaveolens, BUSCO identified 758 (52.7\%) complete orthologs (45.5\% single-copy and $7.2 \%$ duplicated), 260 (18.1\%) fragmented orthologs and $422(29.2 \%)$ missing orthologs. We also found 9178 (13.6\%) genes of E. ivorense and 7203 (10.8\%) genes of $E$. suaveolens with $80 \%$ identity and a $>80 \%$ coverage with the $M$. trunculata protein set. On the other hand, an average realignment of reads back to the reference transcriptome exceeded $95 \%$ in both species (Table 1). All of these statistics indicated the high contiguity, coverage and accuracy of the assembly in both species.

\subsection{Exploratory analysis}

The RBH approach between the two transcriptomes identified 30,374 orthologous contigs between $E$. ivorense and E. suaveolens. The NMDS ordination of the samples based on the expression levels of these orthologs reveal that the first axis separates clearly the two species (Fig. 2). Within each species, samples corresponding to replicates at T0 (control) and at T1 (mild stress) were close to each other while those at T2 (strong stress) displayed more divergent expression patterns that were not congruent between replicates (Fig. 2).

\subsection{Functional annotation of genes}

TransDecoder identified 27,910 (41.38\%) and 27,382 (41.11\%) contigs with coding sequences (ORFs) in E. ivorense and E. suaveolens, respectively. Moreover, 28,589 (42.39\%) and 26,386 (39.61\%) contigs had significant match with the UniProtKB/Swiss-Prot database (E-value threshold $\leq 1 \mathrm{E}-5)$ whereas $16,042(57.47 \%)$ and $14,743(53.84 \%)$ protein sequences contain protein domains of the Pfam database. Furthermore, the Gene Ontology (GO) annotation showed that the two species considerably differ in the number of annotated transcripts with a total of $10,865(10.6 \%)$ and $20,365(21.08 \%)$ transcripts annotated for E. ivorense and E. suaveolens, respectively. However, the three major GO annotation categories were represented by similar percentages in the two species (Fig. 3). In terms of GO categories, 26 GO terms belonged to "Biological process" among which "cellular process" (73.7\%; $67.2 \%)$ and "metabolic process" $(61.5 \%$; $56.5 \%)$ were the most 


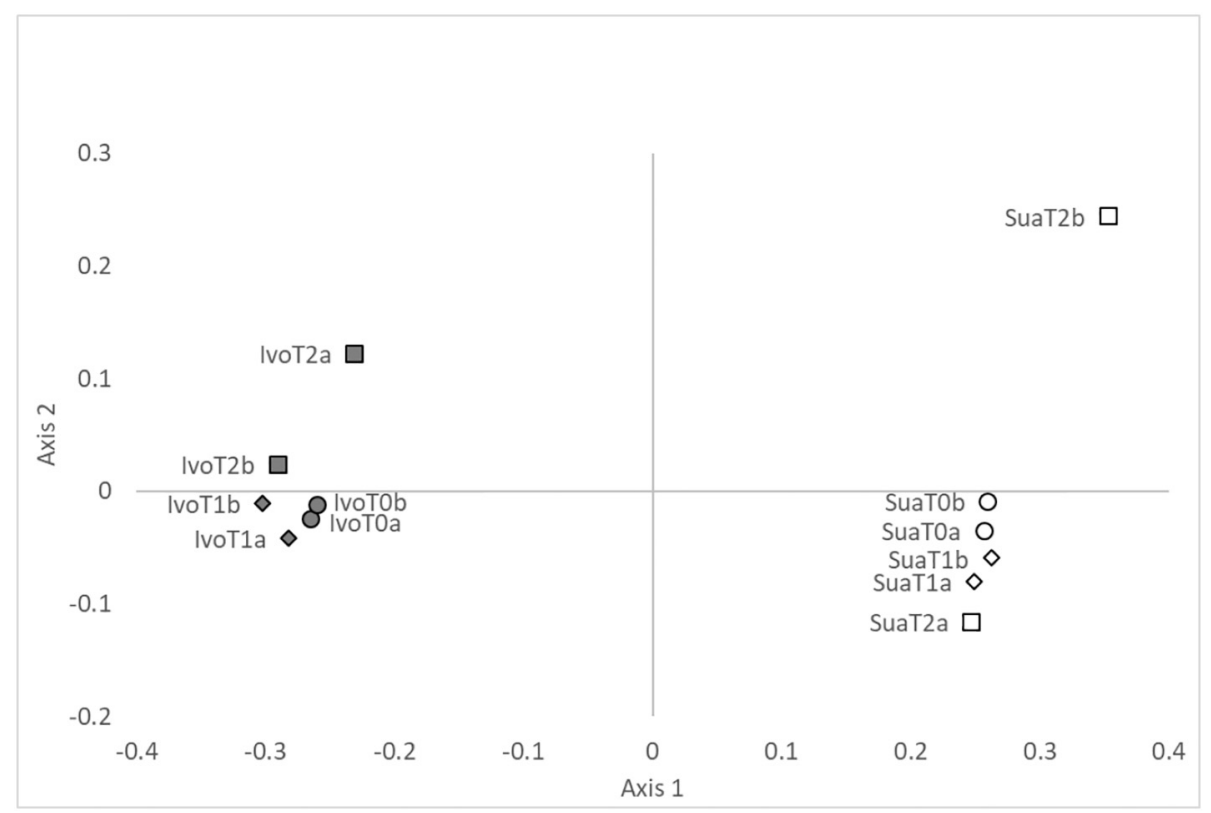

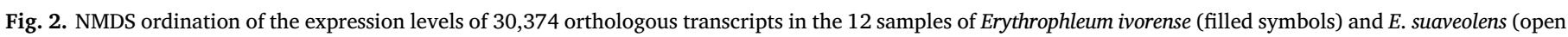

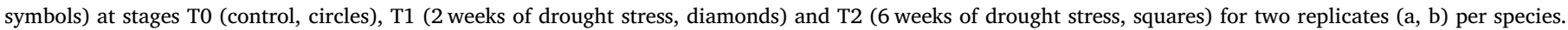

represented GO terms, 14 GO terms belonged to "Molecular function" of which "binding" (60.2\%) and "catalytic activity" (53\%) were the most represented GO terms, and $13 \mathrm{GO}$ terms belonged to "Cellular component" where "cell" (87.9\%; 81\%) and "cell part" (87.9\%; 81\%) were found the most dominant GO terms. Notably, 24 GO terms showed significant gene number differences $(\mathrm{P}<0.001)$ between the two species (Additional file 1). After searching against the eggNOG database, a total of 25,730 and 25,302 protein sequences were assigned to
24 functional categories in E. ivorense and E. suaveolens, respectively. All the identified COG categories were found similarly distributed in both species. The highest number of the contigs were classified in "Function unknown" (26\%), "Signal transduction mechanisms" (12\%), "Posttranslational modification, protein turnover, chaperones" (9\%), "Transcription" (8\%) and "Carbohydrate transport and metabolism" (5\%) (Fig. 4; Additional file 2). Moreover, similar to GO annotation, the two species differed significantly in their KEGG annotation. Using the online
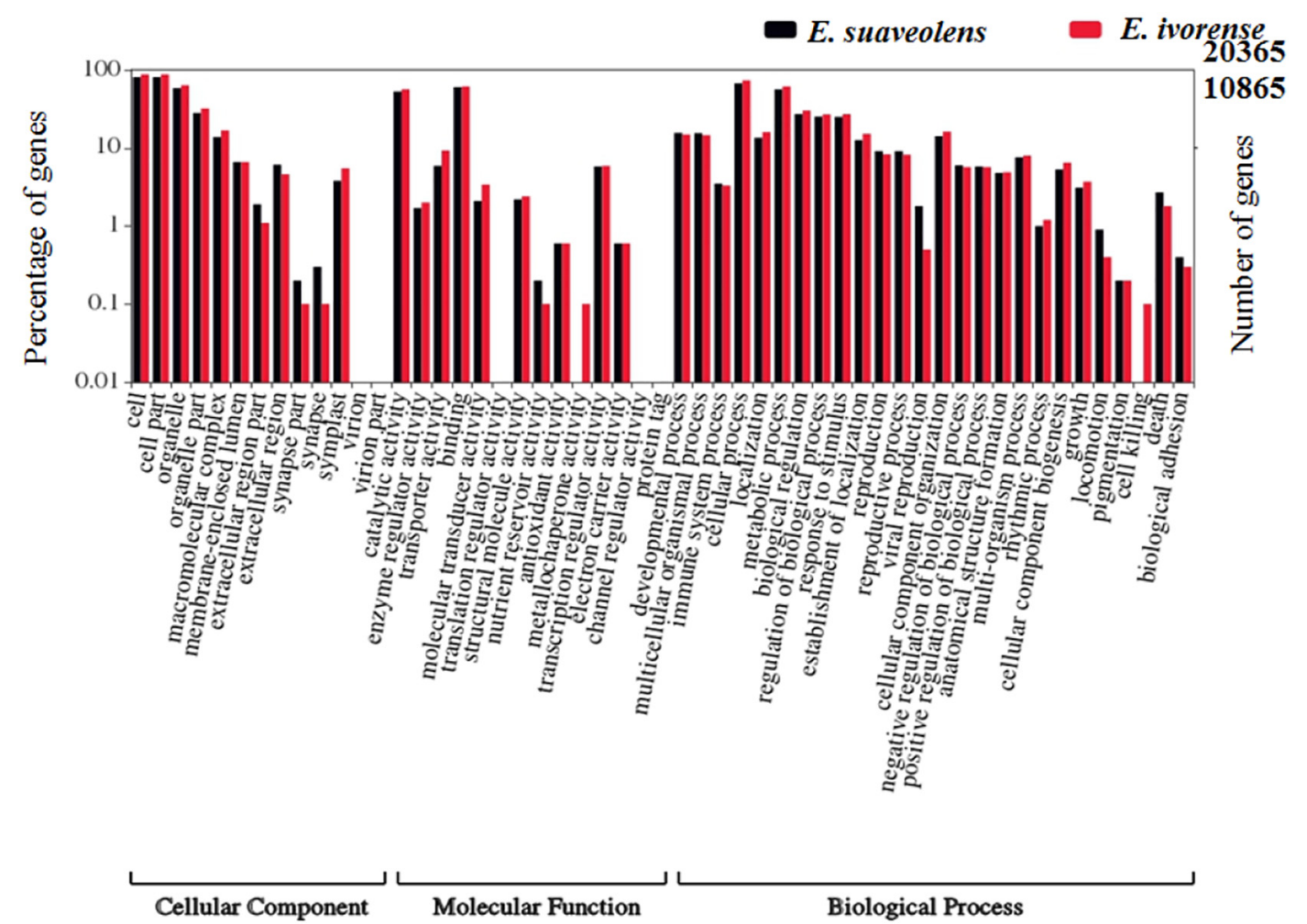

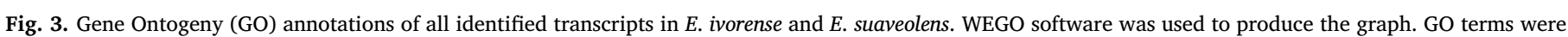

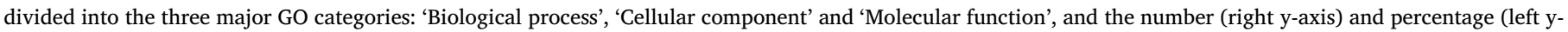
axis) of genes were calculated. 


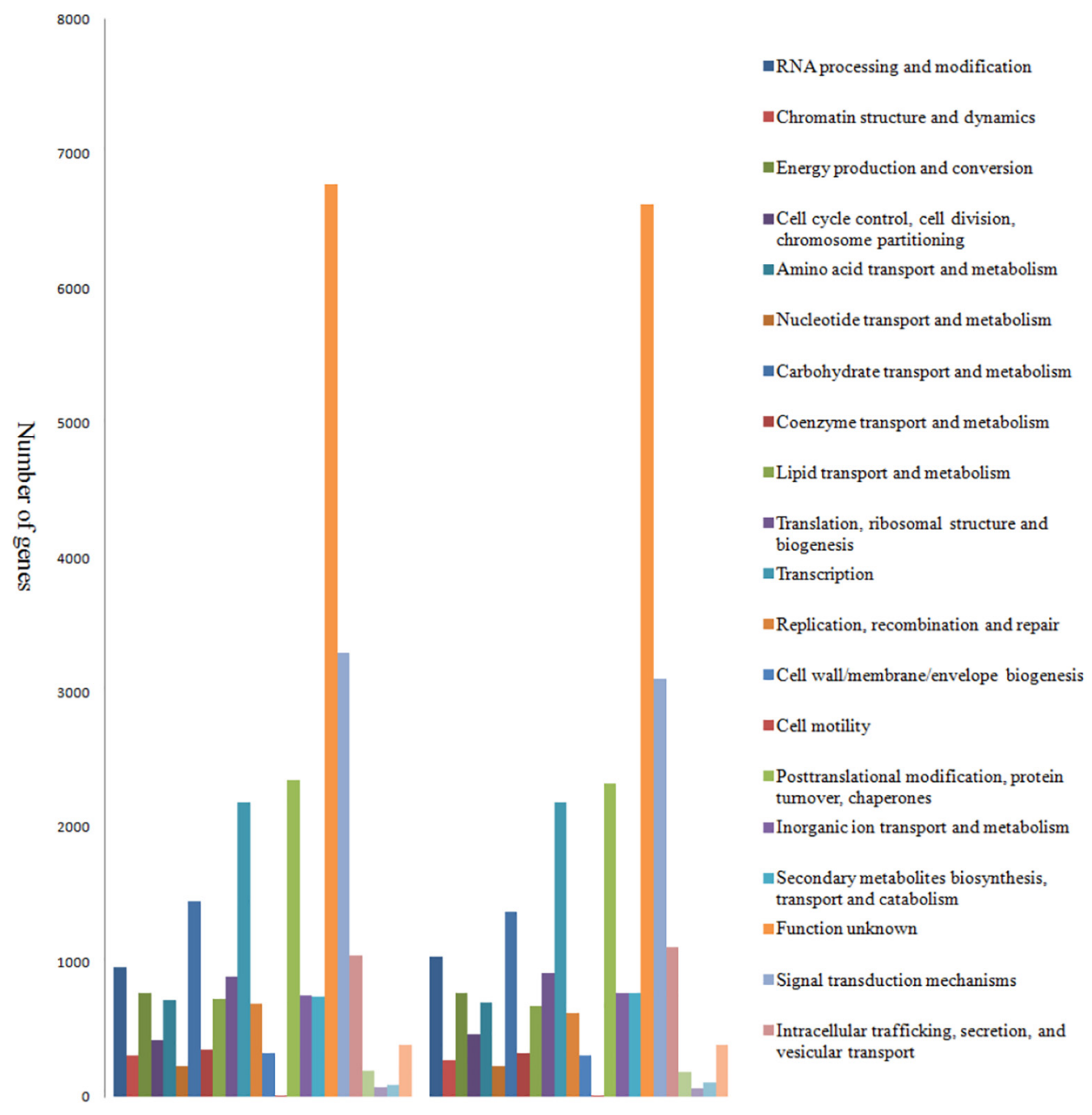

E. ivorense

E. suaveolens

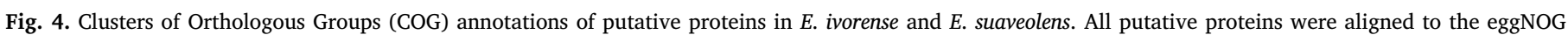
database and were functionally classified into at least 20 functional categories.

KASS server, we identified 10,820 (16.04\%) and 27,994 (42.02\%) KEGG-annotated contigs assigned to 381 and 377 biological pathways in E. ivorense and E. suaveolens, respectively (Additional file 3). The top 10 represented KEGG pathways in both species are shown in Fig. 5.

\subsection{Comparative transcriptome profiling of the two species under drought stress}

To explore the transcriptional response during drought treatments, samples from 2 weeks (T1) and 6 weeks (T2) of stress were compared with their respective controls (T0) and between each other for gene expression differences. From the comparison groups, T0 vs T1, T0 vs T2 and T1 vs T2, the DEG analysis revealed a total of 2020 and 1495 differentially expressed genes (P-value $\geq 0.8$ and $\mid \log 2$ (fold change) $\mid>2$ ) in E. ivorense and E. suaveolens, respectively. Notably, as the drought duration lengthened, an increasing number of gene expression changes were observed in both species. The pattern of expression profiles of overlapping DEGs on each species was visualized through a heat map representing the expression levels for these transcripts at 2 and 6 weeks (Fig. 6). The generated heat map showed that the replicates for each stress stage were well clustered. Clustering analysis showed diverse regulatory patterns of a large proportion of the identified DEGs in response to the drought stages with significant differences in the gene expression profiles between the control, 2 weeks and 6 weeks of stress. Moreover, the expression profiles of 2 weeks stressed plants were grouped closer to those of the control plants than to those of 6 weeks stressed plants in both species (Fig. 6).

Globally, from the change of expression patterns (either up- or down-regulated) of DEGs, it appears that E. suaveolens reacted earlier to drought stress than E. ivorense at the first stage of stress (406 vs 254 DEGs between $\mathrm{T} 0$ and $\mathrm{T} 1$ ), while being relatively less affected following the severe stage of stress (450 vs 1041 DEGs between T1 and T2; Fig. 7A). Remarkably, compared to E. ivorense, E. suaveolens displayed much more up-regulation in response to drought (116 vs 56) at 2 weeks of stress. At the severe stage of stress, the two species showed contrasted expression patterns with a majority of down-regulation in $E$. ivorense and of up-regulation in suaveolens (Fig. 7A). A venn diagram was constructed to further specify commonly and exclusively regulated genes across the three stages of the drought treatment. Notably, most of the drought regulated DEGs seem to be late-response genes in both species (Fig. 7B). Nevertheless, more genes were exclusively differentially expressed at the first stage of stress (T0 to T1) in E. suaveolens (257 vs 158 in E. ivorense) while more genes were exclusively differentially expressed at the late stage of stress (T1 to T2) in E. ivorense (437 vs 237 in E. suaveolens). The venn diagram (Fig. 7B) showed that in both species, T2-T0 and T1-T0 comparisons displayed a relatively small number of shared DEGs, with much higher number of common genes detected in E. suaveolens (93 vs 45 in E. ivorense). However, a much 

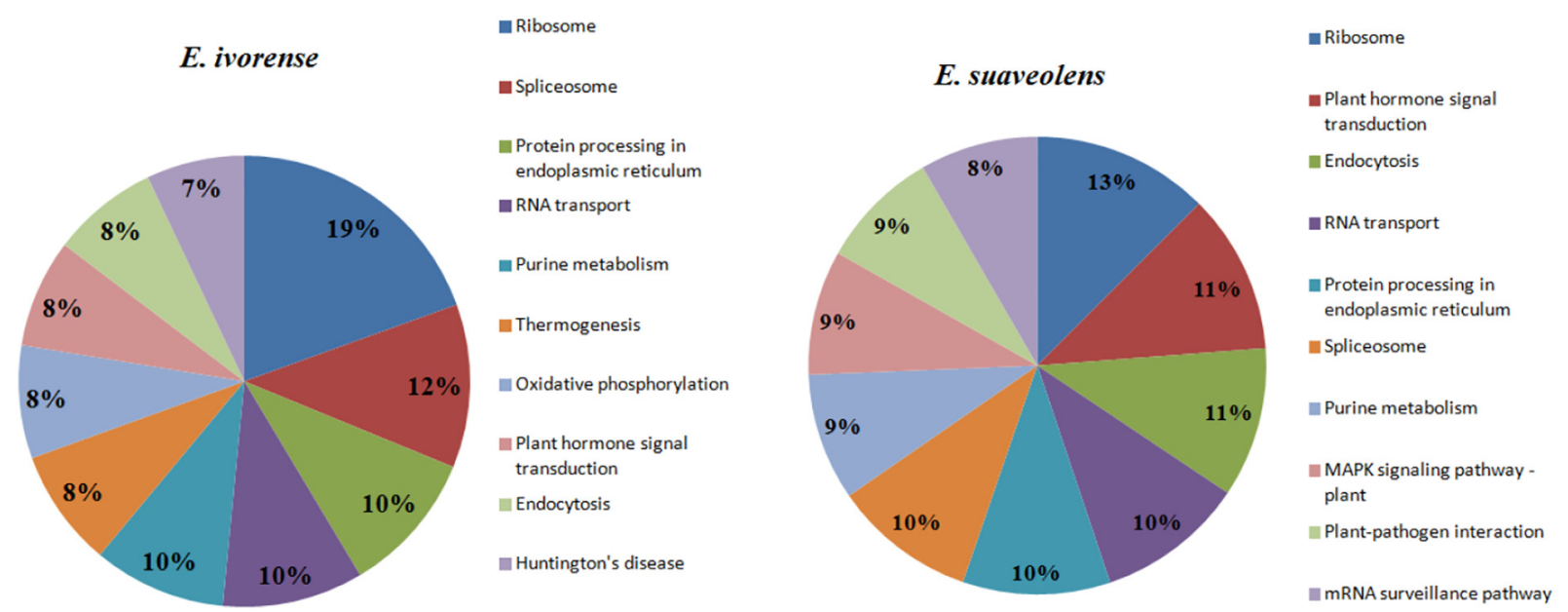

Fig. 5. The percentage of genes identified in the top 10 KEGG (Kyoto Encyclopedia of Genes and Genomes) pathways in E. ivorense and E. suaveolens.

higher number of DEGs were found to be shared between T1-T2 and T0$\mathrm{T} 2$ in E. ivorense compared to E. suaveolens (517 vs 158).

\subsection{Differential expression of orthologs in the two species}

To further investigate the overlap of gene expression profiles in the two species we consider now only the 30,374 identified ortholog genes, among which 1052 were DEGs in one and/or the other species over the drought treatment (T0 vs T2 comparison). From the latter, 587 and 458 DEGs were specific to $E$. ivorense and E. suaveolens, respectively, leaving only seven shared orthologos DEGs in both species (Fig. 7C). Erythrophleum suaveolens displayed more specific DEGs at the early stage of drought (258 vs 73 in E. ivorense), most of them were down-regulated, and there was no shared DEGs (Fig. 7C). At the late stage of stress (T1 vs T2 comparison), more differentially expressed genes were exclusively identified in E. ivorense (464 vs 299). At this stage of drought, the latter species showed a drastic increase in the number of up-regulated DEGs in comparison to the early stage of stress (162 vs 13). However, the number of up-regulated DEGs in E. suaveolens remains always much higher than observed in E. ivorense (255 vs 162).

On the other hand, our results showed that only 7 orthologs DEGs

A

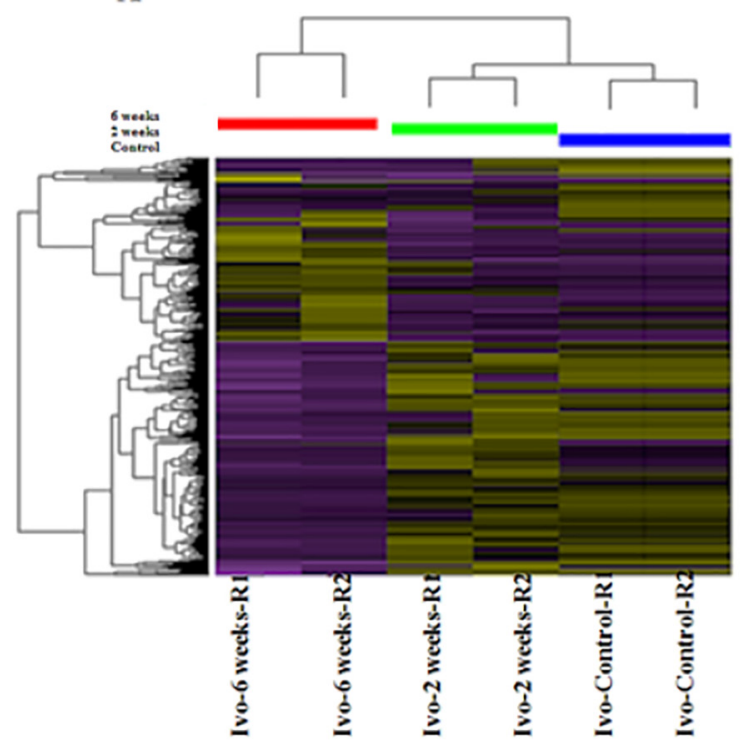

were shared between the two species during the drought treatment. None of them were identified at the early stage of stress. Considering the large proportion of specific DEGs identified in each species, these results indicate that the two species may display different catalogues of genes in response to drought.

\subsection{Functional annotation of DEGs under drought stress}

To speculate the function of the identified DEGs on each species, we applied a GO enrichment analysis using P-value of 0.05 adjusted by false discovery rate (FDR) as the cutoff. Results showed that 289 $(14.3 \%)$ and $398(26.62 \%)$ of DEGs were annotated and assigned to 44 and $46 \mathrm{GO}$ terms in E. ivorense and E. suaveolens, respectively (Fig. 8). In both species, the majority of GO terms fall in the category 'biological process' followed by 'cellular component' and 'molecular function', respectively.

The SEA enrichment analysis of the DEGs that were exclusive to each species indicated possible differences between the two species in reacting to drought at its different stages. At the early stage of drought, the DEGs uniquely up-regulated and down-regulated in E. ivorense were categorized in a small number of GO terms mainly involved in cellular

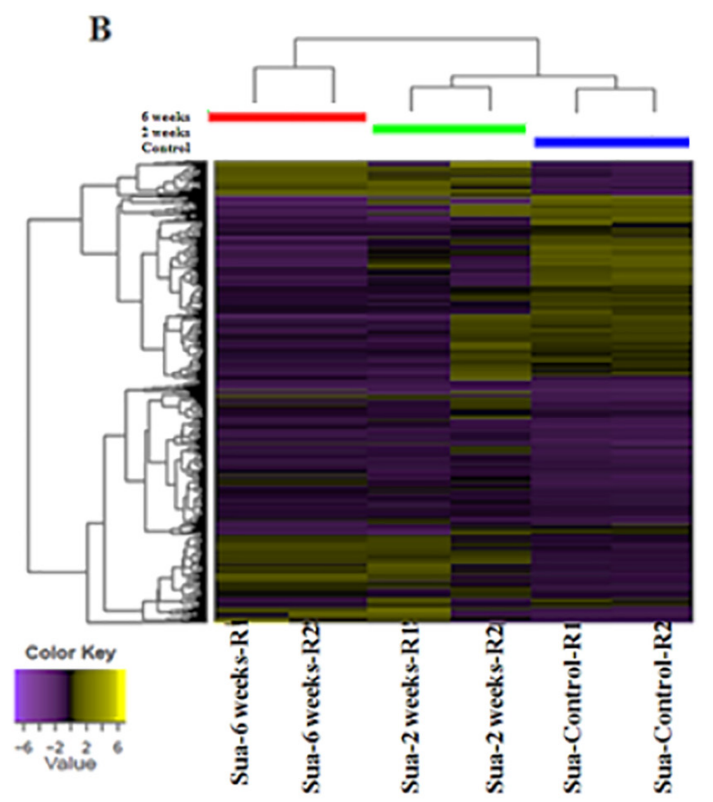

Fig. 6. Hierarchical cluster analysis of DEGs under 2 weeks or 6 weeks of drought stress compared with the control (A: E. ivorense; B: E. suaveolens). 


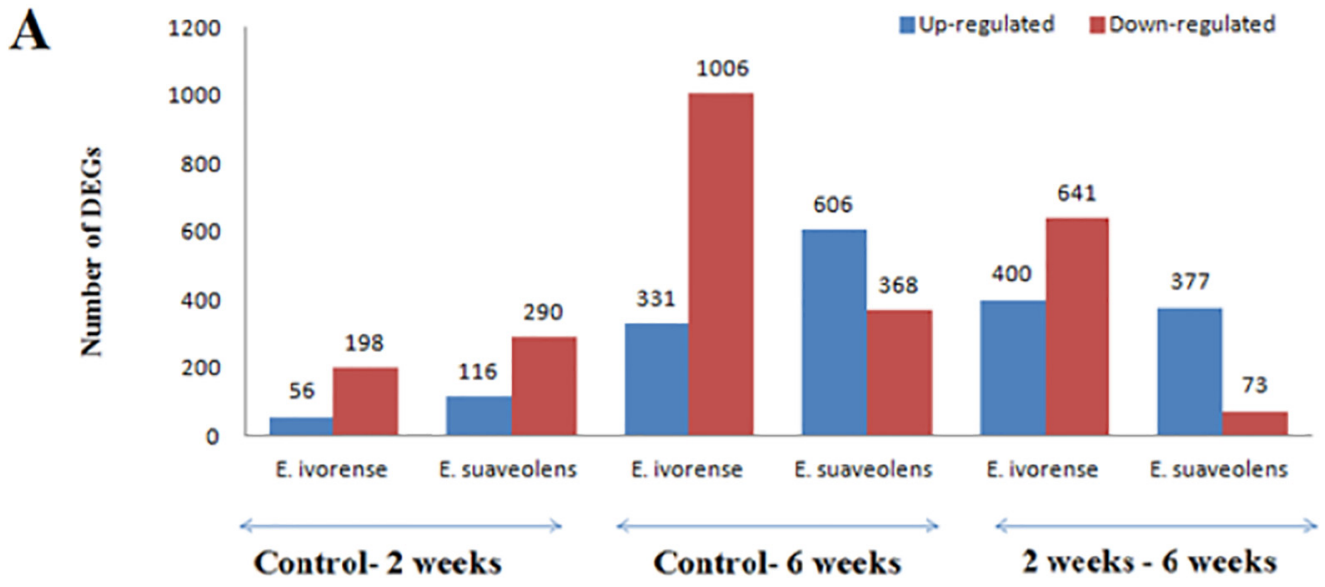

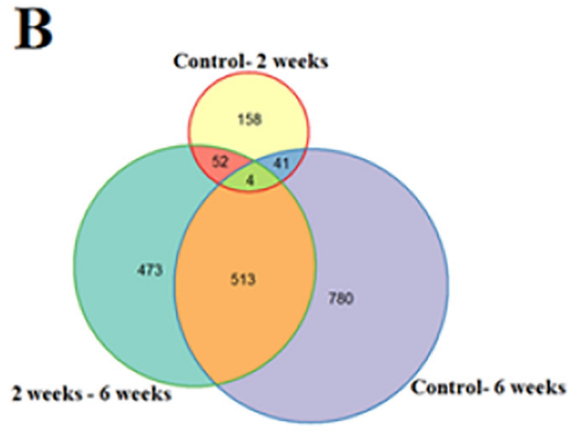

E. ivorense

C

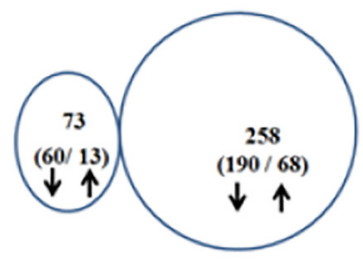

E. Worense $\quad$ E. suaveolens

Control - 2 weeks

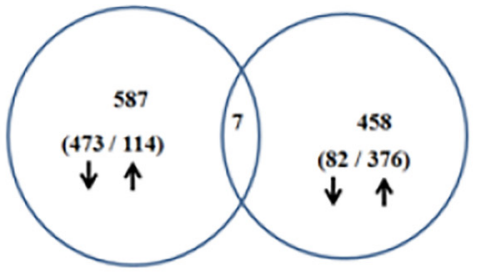

E. ivorense

Control -6 weeks

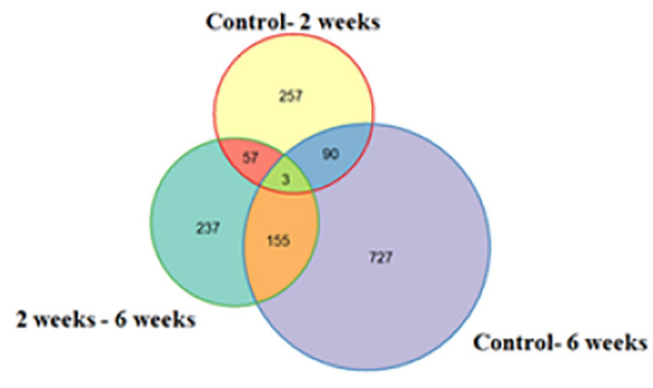

E. suaveolens

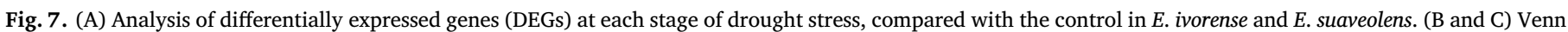

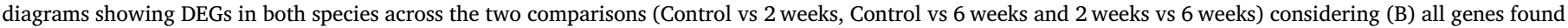

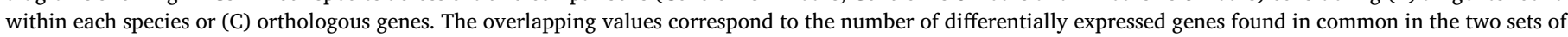
comparisons.

component categories such as 'membrane', 'cell' and 'cell part'. In $E$. suaveolens, we found a large set of greatly enriched up-regulated genes that encode for GO terms involved in signal transduction ('intracellular signaling cascade'; 'signal transduction'; 'iron ion binding'; 'heme binding', 'cation binding') as well as various metabolism-related GO terms ('carbohydrate metabolic process'; 'fructose metabolic process'; 'galactose metabolic process'; 'catalytic activity') and other related to abiotic stress response ('response to abiotic stimulus', 'response to external stimulus'; 'response to stress'). Contrary to E. suaveolens, we noticed that both the up- and the down-regulated genes specific to $E$. ivorense were highly enriched in terms linked to abiotic stresses and involved in signal transduction and metabolic processes only at the late stage of drought (T1 to T2) (Additional file 1). Overall, these results suggest that the two species might employ different genetic pathways to cope with drought at different stages and point toward an earlier response to drought in E. suaveolens compared to E. ivorense.

To identify metabolic pathways or signal transduction pathways displaying significant change in response to drought stress (P value $\leq 0.05$ ), the exclusive and shared DEGs were mapped to terms in the KEGG database (Additional file 4). After 2 weeks of stress, $28.76 \%$ $(21 / 73)$ and $66.66 \%(174 / 258)$ of the specific DEGs identified in $E$. ivorense and E. suaveolens were associated with 20 and 64 KEGG pathways, respectively. In contrast to E. ivorense, in which only one gene encoding for 'Plant hormone signal transduction' was significantly enriched, 61 DEGs (48 down-regulated/13 up-regulated) were found greatly enriched in E. suaveolens. The down-regulated genes were mainly associated with eight metabolic pathways from which photosynthesis (12 DEGs) and glycolysis/gluconeogenesis (6 DEGs) and oxidative phosphorylation (6 DEGs) were the most represented, and three signaling pathways: plant hormone signal transduction (4 DEGs), AMPK signaling pathway (4 DGEs) and calcium signaling pathway (3 DEGs). Similarly, we noticed that almost all the enriched up-regulated genes $(11 / 13)$ were associated with metabolic pathways. At the late stage of drought ( 2 weeks-6 weeks), 50.59\% (297/587) and $27.72 \%$ 

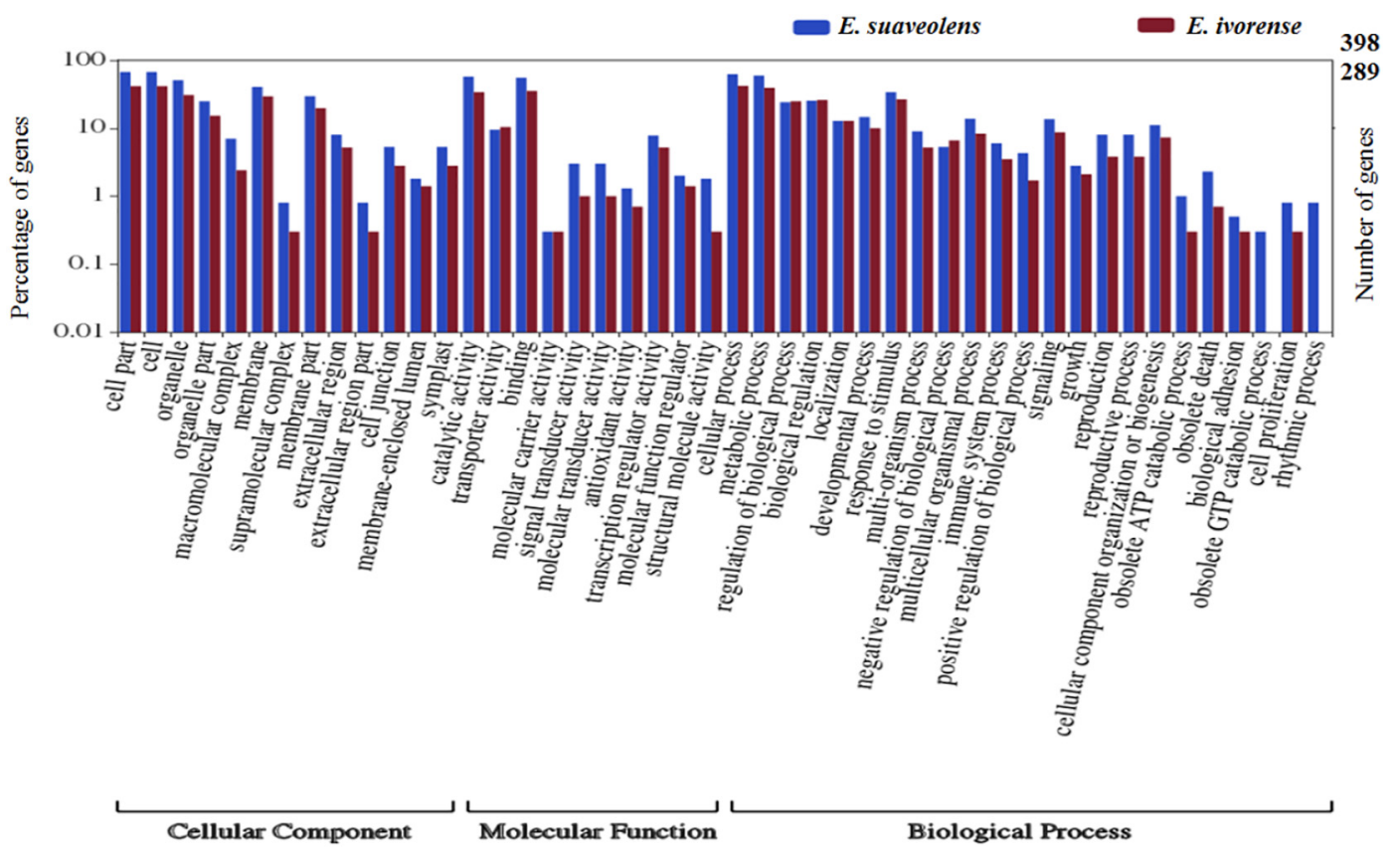

Fig. 8. Gene ontology (GO) annotation of drought-responsive genes in E. ivorense and E. suaveolens.

(127/458) of the exclusive DEGs were associated with 131 and 65 KEGG pathways in E. ivorense and E. suaveolens, respectively. In $E$. ivorense, 62 up-regulated genes were enriched in 24 KEGG pathways among which 'photosynthesis' (8 DEGs), 'glycolysis/gluconeogenesis' (8 DEGs), 'oxidative phosphorylation' (5 DEGs), glyoxylate and dicarboxylate metabolism (5 DEGs), 'mRNA surveillance pathway' (4 DEGs) and 'plant hormone signal transduction' (4 DEGs) were the most represented, while a set of 19 down-regulated genes were found to be enriched in 11 pathways, most of them related to 'metabolism' and 'genetic information processing'. In E. suaveolens, 38 DEGs (24 upregulated and 20 down-regulated) were enriched in 14 pathways mainly associated to metabolic processes. It should be noted that genes encoding for most of the KEGG pathways cited above were found differentially expressed and greatly enriched only at the late stage of drought in E. ivorense and only at the early stage in E. suaveolens.

\subsection{Differential expression of transcription factors during the drought treatment}

A total of 1934 (2.69\%) and $1783(2.67 \%)$ of the assembled unigenes were identified as Transcription factors (TFs) belonging 90 and
87 families in E. ivorense and E. suaveolens, respectively (Additional file $5)$. Except for STAT, HB-PHD and GRF, which were observed only in $E$. ivorense, the remaining TFs families were identified in both species. Moreover, among the top 10 represented TFs families, nine were found common between both species. Among the 1052 differentially expressed orthologs, 15 (6 up-regulated and 9 down-regulated) and 45 (20 up-regulated and 25 down-regulated) differentially expressed TFs belonging to 6 and 19 families were identified following 2 weeks of stress in E. ivorense in E. suaveolens, respectively (Additional file 5). At this stage of drought, we found that most of the differentially expressed TFs in E. suaveolens were categorized into five major families: NAC (6 DEGs), WRKY (5 DEGs), MYB (5 DEGs), bHLH (5 DEGs) and C2H2 (4 DEGs). In E. ivorense, the most represented TFs families were NAC (4 DEGs), WRKY (3 DEGs), MYB (3 DEGs) and bHLH (3 DEGs). At the late stage of stress (T1-T2), much more up-regulated TFs were identified in E. ivorense than E. suaveolens (30 vs 16) (Table 2). Noticeably, among the top ten differentially expressed TFs revealed in E. suaveolens at the early stage of stress, five of them (MYB, bHLH, WRKY, NAC and bZIP) were found the most abundant in $E$. ivorense at the late stage of drought. Interestingly, at this stage of stress, these five TFs families showed more up-regulated expression in E. ivorense than that revealed in E. suaveolens

Table 2

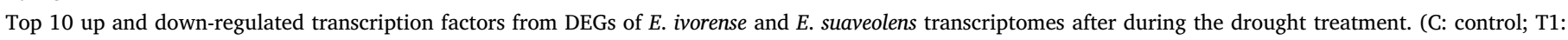
2 weeks; T2: 6 weeks).

\begin{tabular}{|c|c|c|c|c|c|c|c|c|c|c|c|c|}
\hline \multirow{3}{*}{$\mathrm{TF}$ families } & \multicolumn{6}{|c|}{ E. ivorense } & \multicolumn{6}{|c|}{ E. suaveolens } \\
\hline & \multicolumn{2}{|c|}{ C-T1 } & \multicolumn{2}{|c|}{ C-T2 } & \multicolumn{2}{|c|}{$\mathrm{T} 1-\mathrm{T} 2$} & \multicolumn{2}{|c|}{$\mathrm{C}-\mathrm{T} 1$} & \multicolumn{2}{|c|}{ C-T2 } & \multicolumn{2}{|c|}{$\mathrm{T} 1-\mathrm{T} 2$} \\
\hline & Up & Down & Up & Down & Up & Down & Up & Down & Up & Down & Up & Down \\
\hline WRKY & 1 & 2 & 4 & 3 & 3 & 1 & 3 & 2 & 5 & 3 & 2 & 1 \\
\hline MYB & 1 & 2 & 3 & 2 & 3 & 2 & 3 & 2 & 3 & 3 & 1 & 0 \\
\hline bHLH & 1 & 2 & 5 & 1 & 3 & 2 & 2 & 3 & 3 & 3 & 0 & 1 \\
\hline NAC & 3 & 1 & 4 & 3 & 3 & 1 & 3 & 3 & 4 & 3 & 1 & 1 \\
\hline $\mathrm{C} 3 \mathrm{H}$ & 0 & 0 & 3 & 0 & 3 & 0 & 1 & 2 & 2 & 1 & 0 & 1 \\
\hline $\mathrm{C} 2 \mathrm{H} 2$ & 0 & 0 & 1 & 2 & 1 & 2 & 1 & 3 & 5 & 1 & 1 & 1 \\
\hline AP2/ERF-ERF & 0 & 0 & 1 & 1 & 1 & 1 & 1 & 2 & 2 & 2 & 1 & 1 \\
\hline GRAS & 0 & 0 & 0 & 2 & 0 & 2 & 0 & 0 & 1 & 0 & 1 & 0 \\
\hline bZIP & 0 & 1 & 4 & 1 & 3 & 1 & 1 & 0 & 0 & 0 & 0 & 0 \\
\hline mTERF & 0 & 1 & 1 & 0 & 0 & 0 & 0 & 1 & 0 & 1 & 0 & 0 \\
\hline
\end{tabular}


(15 DEGs vs 4 DEGs) (Table 2).

\section{Discussion}

Water availability is a critical driver of the geographical distribution and abundance of plant species (Kunstler et al., 2016), which could explain the parapatric distribution of sister Erythrophleum species: $E$. ivorense is distributed in evergreen rainforests, whereas E. suaveolens is found in semi-evergreen and in gallery forests under more seasonal and/or drier climates (Duminil et al., 2013), suggesting that it might be more adapted to survive drought stress. Our RNA-Seq based comparative transcriptomics analysis of seedlings is a first attempt to unravel the potential molecular mechanisms underlying the differences in response to drought in these species.

Our study focused on analyzing differential gene expression from the early stage of wilting of the seedlings due to the important roles of early wilting-responsive genes in mediating the effects of drought stress (Das et al., 2012). Our results showed that, compared to E. ivorense, E. suaveolens presented earlier signs of wilting, which suggest that the latter species displayed earlier morphological response and probably earlier physiological and molecular responses to drought.

Our transcriptomes generated from Trinity assembly showed similar characteristics in both species, with N50 and the mean length of the contigs being comparable to those reported in many Fabaceae species (e.g., Wegrzyn et al., 2016; Wong et al., 2011) and very similar G/C content $(41 \%)$, consistent with observations in other closely related species (Herraiz et al., 2016). On the other hand, although displaying similar distributions of GO terms and KEGG pathways, the two species differed greatly in the number of annotated transcripts in these two databases. This is probably due to the differences in the amount of sequencing data generated from the two species and maybe due to the lack of available genome resources in the subfamily Caesalpinoideae. While we identified orthologs between the two species for nearly half of the contigs found within each species, the expression patterns of these orthologs for the 12 samples studied differed more between species than between treatments within each species (Fig. 2). Hence, despite their phylogenetic proximity, the two species display distinct gene expression patterns.

When exposed to drought stress, plants display various regulatory mechanisms coupled with extensive gene expression changes (up-regulation and down-regulation; Shanker et al., 2014; Fox et al., 2017). In some model plants such as Arabidopsis thaliana (Matsui et al., 2008), Gossypium hirsutum (Chen et al., 2013), Medicago truncatula (Zhang et al., 2014) and Oryza sativa (Todaka et al., 2017), much more upregulation was observed under drought stress. Similar patterns were also reported for some tropical and non-tropical tree species relatively adapted to drought, belonging to diverse botanical families and occurring across heterogeneous environments such as Tectona grandis (Lamiaceae; Tripathi et al., 2017), Populus davidiana (Salicaceae; Mun et al., 2017), Prosopis juliflora (Mimosaceae; George et al., 2017) and Coffea canephora (Rubiaceae; de Freitas Guedes et al., 2018). In contrast, much more down-regulation was observed in Quercus lobata (Fagaceae; Gugger et al., 2017) and Pinus halepensis (Pinaceae; Fox et al., 2017) endemic to California and Mediterranean basin, respectively. In our results, when the two species were analyzed separately, we found that $E$. ivorense displayed more differentially expressed genes under drought stress (2020 vs 1495 ), whereas E. suaveolens was characterized by higher rate of up-regulation $(62.21 \%$ vs $24.75 \%)$ over the duration of the drought treatment, being more evident at the late stage of drought. We also noticed that almost all the DEGs for which we found orthologs were exclusive to one of the two species.

Functional enrichment analyses of these DEGs provided clues to the molecular mechanisms involved in drought response. Our results revealed a large number of GO terms and KEGG pathways that may be involved in the tolerance to diverse biotic and abiotic constraints. In agreement with our results, several recent studies have demonstrated that the crosstalk among signaling and metabolic pathways plays a key role in stress signaling and drought tolerance (Golldack et al., 2014; de Zelicourt et al., 2016; Dong et al., 2017; Zhu, 2016). The early drought response is triggered by the repression or the induction of genes encoding primary osmotic stress signals (e.g Ca2 + and MAPK signaling) or by secondary signal metabolites (e.g. ABA, ethylene, sugars), generally in a transient mode (Chaves et al., 2003; Chinnusamy et al., 2004; Huang et al., 2012). Moreover, it has been reported that the downregulation of some important metabolic pathways such as photosynthesis, oxidative phosphorylation and glycolysis is the primary acclimation response to drought stress. These pathways as well as other involved in compatible solutes (e.g. proline, polyamines, trehalose) and secondary metabolites (e.g. flavonoids, monobactam) biosynthesis play a crucial role in controlling the drought response in plants (Krasensky and Jonak, 2012; Zhang and Xiao, 2018). Indeed, according to Cuin and Shabala (2007), the early accumulation of antioxidants, soluble sugars, oligosaccharides and amino acids, resulting from increased protein degradation, enhances the drought tolerance since these latter may act as osmolytes or reactive oxygen species (ROS) scavengers. Our pathways enrichment analysis showed that, in contrast to E. ivorense, an extensive down-regulation of genes associated to photosynthesis (12 DEGs), glycolysis/gluconeogenesis (6 DEGs) and oxidative phosphorylation (6 DEGs) and a considerable up- and down-regulation of genes encoding for some amino acid (Tryptophan) and secondary metabolites biosynthesis were highly enriched at the early stage of drought in $E$. suaveolens. On the other hand, the GO enrichment analysis revealed a large set of down and up GO terms related to response to stress and stimuli as well as some cellular processes. These results may, in part, explain the early wilting symptoms observed in E. suaveolens, as a first morphological reaction against water shortage. In fact, it has been demonstrated that plants react to drought by a reduction in photosynthetic activity caused by a small decline in stomatal conductance, which may have protective effects against stress, by allowing plant water saving and improving plant water-use efficiency. Taken together, these findings suggest an earlier response to drought compared to $E$. ivorense. In agreement, previous studies have concluded that photosynthesis, carbohydrate metabolism and cell division (Nelissen et al., 2018) are generally down-regulated under early drought stress to maintain normal metabolic activities under dehydration (HayanoKanashiro et al., 2009). At the late stage of drought (T1-T2), an overall similar GO and KEGG pathways enrichment pattern was observed in $E$. suaveolens, which implies that the main response to drought at the molecular level in this species occurred at the early stage of stress. The early down-regulation of the metabolic pathways could be a strategy of a prompt response to stress allowing to sustain the metabolism even under severe drought conditions. At the late stage of drought (T1-T2), we noticed a further enrichment of down and up associated with diverse metabolic pathways as well as numerous GO related response to stress and biological processes in E. suaveolens, which implies that the response to drought in this species starts early and become stronger with the intensification of water deficit. However, a noticeable enrichment of many up-regulated genes involved in signaling and metabolic pathways as well as a large set of GO terms associated many "Biological process" and "cellular process" categories was revealed in $E$. ivorense at the late stage of drought. Such pattern suggests that the latter species presents a slow and sequential molecular changes and a gradual preparation of adaptation to drought and thus a late response to drought compared to E. suaveolens. In agreement, recent reports have suggested that the up-regulation of genes encoding $\mathrm{Ca}_{2}{ }^{+}$, MAPK signaling as well as some metabolic pathways, including photosynthesis, glycolysis and biosynthesis of secondary metabolites and lipids, at the mild stage of drought confers drought tolerance in Pennisetum glaucum (Poaceae) (Dudhate et al., 2018) and Ammopiptanthus mongolicus (Fabaceae) (Gao et al., 2015). Interestingly, given the very small number of shared DEGs, the contrasted differential expression profiles (down and up-regulation) observed in the two species along the drought treatment 
suggests that each of them uses its exclusive catalogue of genes to activate similar signaling and metabolism-related pathways to respond to drought at different stages of stress. These findings suggest that one difference between $E$. ivorense and $E$. suaveolens could be the rapidness of drought perception and the modulation of the pathways involved in drought response. On the other hand, we noticed that E. suaveolens displayed more up-regulated genes than $E$. ivorense over the drought treatment. Assuming that a large part of these genes may be encoding for signaling and metabolic pathways but not unannotated using GO and KEGG databases from E. suaveolens transcriptome, we suggest that these sets of up-regulated genes could be involved in the response to drought at its different stages and may confer better drought tolerance to this species. This is in line with previous comparative studies performed in Oryza sativa (Poaceae) (Lenka et al., 2011) and Trifolium pratense (Fabaceae) (Yates et al., 2014) genotypes and as well as two Corchorus species (Tiliaceae) (Yang et al., 2017) and two tallgrass species (Hoffman and Smith, 2018) with contrasting drought tolerance in which an increased number of up-regulated genes encoding for signaling and metabolism-related pathways was observed in the tolerant genotypes.

Several studies have highlighted the key role of transcription factors (TF) in mediating drought tolerance in plants, including tree species (Rabara et al., 2015; Joshi et al., 2016; Mun et al., 2017). Some of these studies have demonstrated that TFs are involved in diverse physiological and molecular mechanisms, including stomata and chloroplast development, signaling, plant growth regulation and various metabolic and hormonal pathways. In the present study, a comparable number of TFs and TFs families were identified in $E$. ivorense and $E$. suaveolens. Interestingly, MYB, C2H2, bHLH, WRKY, NAC, bZIP and C3H, which are considered among the major families associated to the drought responses in some model plants (Moumeni et al., 2011; Pereira et al., 2011) were identified among the most represented TFs families in both species. According to Golldack et al. (2014), the network between these TFs families plays a major role in $\mathrm{ABA}$ and $\mathrm{Ca}_{2}{ }^{+}$signaling by controlling the intracellular ROS accumulation as well as some epigenetic processes such as DNA methylation under drought stress. Moreover, recent studies have demonstrated that the rapid up-regulation of genes encoding these TFs under drought may enhance drought tolerance in rice (Hong et al., 2016) and Populus species (Mun et al., 2017). In our data, this pattern is especially in line in E. suaveolens at the early stage of drought treatment and only at the severe drought treatment in $E$. ivorense in the ten top TFs identified in both species (Table 2). Overall, the analysis of the differential expression of TFs during drought stress was in agreement with the overall pattern of gene expression profile observed in both species. Such pattern confirms that, compared to $E$. ivorense, E. suaveolens may exhibit an early response to drought by initiating an extensive transcriptional regulation that enhance the crosstalk among the diverse signaling, hormonal and metabolic pathways involved in the regulation of its drought response.

\section{Conclusion}

The present study represents a first attempt in using the RNA-seq approach to unravel the genetic basis of drought stress response in $E$. ivorense and E. suaveolens, two sister tree species characterized by contrasting geographical distribution and ecological amplitude in the African rainforests. The high quality and comprehensiveness of the de novo assembled transcriptomes generated for both species allowed the identification of a large number of DEGs implicated in diverse signaling and metabolic pathways associated with drought tolerance. Our phenotypic results revealed that $E$. suaveolens seedlings seem to suffer earlier from drought than $E$. ivorense seedlings but this trend reverted at a later stage of drought. Our transcriptome data showed also, in the two studied species, the number of exclusive DEGs far exceeded the number of shared DEGs, suggesting that each species develops a specific response to drought. The analysis of GO terms and KEGG pathways retrieved from DEGs as well as from differentially expressed TFs suggests that E. suaveolens may present an early response to drought probably governed by the down-regulation of the major metabolic pathways and TFs, which could allow the species to maintain only the sufficient requirements for a normal metabolism under drought stress. In contrast, $E$. ivorense seems to display a late response to drought strengthened by the up-regulation of some TFs and signaling and metabolism-related pathways only at the severe stage of drought. Given the very small number of shared DEGs, the patterns observed suggest that each of the study species employs its own catalogue of genes to modulate drought using similar signaling and metabolic pathways but at different phases. The present study provides valuable insights on the genetic basis of drought response in E. ivorense and E. suaveolens. Understanding these mechanisms could have important implications to predict the impact of ongoing and future climate change on African rainforests, as drought events are projected to become more severe in tropical regions, including African rainforests. As RNA-seq approach becomes more accessible in ecological studies, the transcriptome resources as well as the obtained results could also serve as a reference for further work on the genetic basis of abiotic stresses in these two species and other tropical tree species.

Supplementary data to this article can be found online at https:// doi.org/10.1016/j.gene.2019.01.027.

\section{Conflict of interest}

The authors have no conflicts of interest to declare.

\section{Acknowledgements}

We thank Wallonie-Bruxelles International (WBI) for the provided Postdoctoral Fellowships attributed to M.N. The genetic analyses were funded by the F.R.S. - FNRS (grants $n^{\circ}$ J.0292.17F and T.0163.13) and the Belgian Science Policy Office (Belspo, project AFRIFORD). Computational resources have been provided by the Consortium des Équipements de Calcul Intensif (CÉCI), funded by the Fonds de la Recherche Scientifique de Belgique (F.R.S. - FNRS) under Grant No. 2.5020.11. We would like also to thank Dr. Marie Baucher (FNRS-FRS) and an anonymous referee for their constructive comments on previous versions of the manuscript.

\section{References}

Akoègninou, A., Van der Burg, W.J., Van der Maesen, L.J.G., 2006. Flore analytique du Bénin (No. 06.2). Backhuys Publishers.

An, Miao, Deng, Min, Zheng, Si-Si, Song, Yi-Gang, 2016. De novo transcriptome assembly and development of SSR markers of oaks Quercus Austrocochinchinensis and Q. Kerrii (Fagaceae). Tree Genet. Genomes 12 (6), 103.

Baldo, Laura, Emília Santos, M., Salzburger, Walter, 2011. Comparative transcriptomics of Eastern African cichlid fishes shows signs of positive selection and a large contribution of untranslated regions to genetic diversity. Genome Biol. Evol. 3, 443-455.

Bolger, Anthony M., Lohse, Marc, Usadel, Bjoern, 2014. Trimmomatic: a flexible trimmer for Illumina sequence data. Bioinformatics 30 (15), 2114-2120.

Bonal, D., Burban, B., Stahl, C., Wagner, F., 2016. The response of tropical rainforests to drought-lessons from recent research and future prospects. Ann. For. Sci. 73 (1), $27-44$.

Buytaert, W., Cuesta-Camacho, F., Tobón, C., 2011. Potential impacts of climate change on the environmental services of humid tropical alpine regions. Glob. Ecol. Biogeogr 20 (1), 19-33.

Camacho, Christiam, Coulouris, George, Avagyan, Vahram, Ma, Ning, Papadopoulos, Jason, Bealer, Kevin, Madden, Thomas L., 2009. BLAST +: architecture and applications. BMC Bioinf. 10 (1), 421.

Chaves, Manuela M., Maroco, Joao P., Pereira, Joao S., 2003. Understanding plant responses to drought-from genes to the whole plant. Funct. Plant Biol. 30 (3), 239-264.

Chen, Yun, Liu, Zhi-Hao, Feng, Li, Zheng, Yong, Li, Deng-Di, Li, Xue-Bao, 2013. Genomewide functional analysis of cotton (Gossypium Hirsutum) in response to drought. PLoS One 8 (11), e80879.

Chinnusamy, Viswanathan, Schumaker, Karen, Zhu, Jian-Kang, 2004. Molecular genetic perspectives on cross-talk and specificity in abiotic stress signalling in plants. J. Exp. Bot. 55 (395), 225-236.

Corlett, Richard T., 2016. Plant diversity in a changing world: status, trends, and 
conservation needs. Plant Divers. 38 (1), 10-16.

Cuin, Tracey Ann, Shabala, Sergey, 2007. Compatible solutes reduce ROS-induced potassium efflux in Arabidopsis roots. Plant Cell Environ. 30 (7), 875-885.

Das, Akan, Das, Sudripta, Mondal, Tapan Kumar, 2012. Identification of differentially expressed gene profiles in young roots of tea [Camellia Sinensis (L.) O. Kuntze] subjected to drought stress using suppression subtractive hybridization. Plant Mol. Biol. Report. 30 (5), 1088-1101.

Dong, Yanpeng, Fan, Guoqiang, Zhao, Zhenli, Xu, Enkai, Deng, Minjie, Wang, Limin, Niu, Suyan, 2017. Transcriptome-wide profiling and expression analysis of two accessions of Paulownia Australis under salt stress. Tree Genet. Genomes 13 (5), 97.

Du, Zhou, Zhou, Xin, Ling, Yi, Zhang, Zhenhai, Su, Zhen, 2010. AgriGO: a GO analysis toolkit for the agricultural community. Nucleic Acids Res. 38 (suppl 2), W64-W70.

Dudhate, Ambika, Shinde, Harshraj, Tsugama, Daisuke, Liu, Shenkui, Takano, Tetsuo, 2018. Transcriptomic analysis reveals the differentially expressed genes and pathways involved in drought tolerance in pearl millet [Pennisetum glaucum (L.) R. Br]. PLoS One 13 (4), e0195908.

Duminil, Jérôme, Myriam, Heuertz, Doucet, J.-L., Bourland, Nils, Cruaud, Corinne, Gavory, Fréderick, Doumenge, Charles, Navascués, Miguel, Hardy, Olivier J., 2010. CpDNA-based species identification and phylogeography: application to African tropical tree species. Mol. Ecol. 19 (24), 5469-5483.

Duminil, Jerome, Brown, Richard P., Ewédjè, Eben-Ezer B.K., Mardulyn, Patrick, Doucet, Jean-Louis, Hardy, Olivier J., 2013. Large-scale pattern of genetic differentiation within African rainforest trees: insights on the roles of ecological gradients and past climate changes on the evolution of Erythrophleum Spp (Fabaceae). BMC Evol. Biol. 13 (1), 195.

Duminil, Jérôme, Mona, Stefano, Mardulyn, Patrick, Doumenge, Charles, Walmacq, Frédéric, Doucet, Jean-Louis, Hardy, Olivier J., 2015. Late Pleistocene molecular dating of past population fragmentation and demographic changes in African rain forest tree species supports the forest refuge hypothesis. J. Biogeogr. 42 (8), $1443-1454$.

Duveiller, Gregory, Defourny, Pierre, Desclée, Baudouin, Mayaux, P., 2008. Deforestation in Central Africa: estimates at regional, national and landscape levels by advanced processing of systematically-distributed Landsat extracts. Remote Sens. Environ. 112 (5), 1969-1981.

Elmer, Kathryn R., Shaohua, Fan, Gunter, H.M., Jones, J.C., Boekhoff, S., Kuraku, Shigehiro, Meyer, Axel, 2010. Rapid evolution and selection inferred from the transcriptomes of sympatric crater lake cichlid fishes. Mol. Ecol. 19, 197-211.

Finn, Robert D., Clements, Jody, Eddy, Sean R., 2011. HMMER web server: interactive sequence similarity searching. Nucleic Acids Res. 39 (suppl_2), W29-W37.

Finn, Robert D., Coggill, Penelope, Eberhardt, Ruth Y., Eddy, Sean R., Mistry, Jaina, Mitchell, Alex L., Potter, Simon C., Punta, Marco, Qureshi, Matloob, SangradorVegas, Amaia, 2015. The Pfam protein families database: towards a more sustainable future. Nucleic Acids Res. 44 (D1), D279-D285.

Fox, Hagar, Doron-Faigenboim, Adi, Kelly, Gilor, Bourstein, Ronny, Attia, Ziv, Zhou, Jing, Moshe, Yosef, Moshelion, Menachem, David-Schwartz, Rakefet, 2017. Transcriptome analysis of Pinus Halepensis under drought stress and during recovery. Tree Physiol. $1-19$.

Fracasso, Alessandra, Trindade, Luisa M., Amaducci, Stefano, 2016. Drought stress tolerance strategies revealed by RNA-Seq in two Sorghum genotypes with contrasting WUE. BMC Plant Biol. 16 (1), 115.

de Freitas Guedes, Fernanda Alves, Nobres, Priscilla, Ferreira, Daniela Cristina Rodrigues, Menezes-Silva, Paulo Eduardo, Ribeiro-Alves, Marcelo, Correa, Régis Lopes, DaMatta, Fábio Murilo, Alves-Ferreira, Márcio, 2018. Transcriptional memory contributes to drought tolerance in coffee (Coffea Canephora) plants. Environ. Exp. Bot. 147 220-233.

Fu, Limin, Niu, Beifang, Zhu, Zhengwei, Wu, Sitao, Li, Weizhong, 2012. CD-HIT: accelerated for clustering the next-generation sequencing data. Bioinformatics 28 (23), $3150-3152$

Gao, Fei, Wang, Jianyue, Wei, Shanjun, Li, Zhanglei, Wang, Ning, Li, Huayun, Feng, Jinchao, Li, Hongjie, Zhou, Yijun, Zhang, Feixiong, 2015. Transcriptomic analysis of drought stress responses in Ammopiptanthus Mongolicus leaves using the RNA-Se technique. PLoS One 10 (4), e0124382.

Gao, Fei, Li, Huayun, Xiao, Zihua, Wei, Chunxiang, Feng, Jinchao, Zhou, Yijun, 2018. De novo transcriptome analysis of Ammopiptanthus Nanus and its comparative analysis with a. Mongolicus. Trees 32 (1), 287-300.

George, Suja, Manoharan, Deepeka, Li, Jie, Britton, Monica, Parida, Ajay, 2017. Transcriptomic responses to drought and salt stress in desert tree Prosopis Juliflora. Plant Gene 12, 114-122.

Golldack, Dortje, Li, Chao, Mohan, Harikrishnan, Probst, Nina, 2014. Tolerance to drought and salt stress in plants: unraveling the signaling networks. Front. Plant Sci. 5,151 .

Gugger, Paul F., Peñaloza-Ramírez, Juan Manuel, Wright, Jessica W., Sork, Victoria L. 2017. Whole-transcriptome response to water stress in a California endemic oak, Quercus Lobata. Tree Physiol. 37 (5), 632-644.

Haas, Brian J., Papanicolaou, Alexie, Yassour, Moran, Grabherr, Manfred, Blood, Philip D., Bowden, Joshua, Couger, Matthew Brian, Eccles, David, Li, Bo, Lieber, Matthias, 2013. De novo transcript sequence reconstruction from RNA-Seq using the trinity platform for reference generation and analysis. Nat. Protoc. 8 (8), 1494.

Hayano-Kanashiro, C., Calderón-Vázquez, C., Ibarra-Laclette, E., Herrera-Estrella, L., Simpson, J., 2009. Analysis of gene expression and physiological responses in three Mexican maize landraces under drought stress and recovery irrigation. PLoS one 4 (10), e7531.

Herraiz, Francisco J., Blanca, José, Ziarsolo, Pello, Gramazio, Pietro, Plazas, Mariola, Anderson, Gregory J., Prohens, Jaime, Vilanova, Santiago, 2016. The first de novo transcriptome of pepino (Solanum muricatum): assembly, comprehensive analysis and comparison with the closely related species $S$. caripense, potato and tomato. BMC
Genomics 17 (1), 321.

Hoffman, Ava M., Smith, Melinda D., 2018. Gene expression differs in codominant prairie grasses under drought. Mol. Ecol. Resour. 18 (2), 334-346.

Hong, Yongbo, Zhang, Huijuan, Huang, Lei, Li, Dayong, Song, Fengming, 2016. Overexpression of a stress-responsive NAC transcription factor gene ONAC022 improves drought and salt tolerance in Rice. Front. Plant Sci. 7, 4.

Huang, Guo-Tao, Ma, Shi-Liang, Bai, Li-Ping, Zhang, Li, Ma, Hui, Jia, Ping, Liu, Jun, Zhong, Ming, Guo, Zhi-Fu, 2012. Signal transduction during cold, salt, and drought stresses in plants. Mol. Biol. Rep. 39 (2), 969-987.

Huang, L.K., Yan, H.D., Zhao, X.X., Zhang, X.Q., Wang, J., Frazier, T., Yin, G., Huang, X., Yan, D.F., Zang, W.J., 2015. Identifying differentially expressed genes under heat stress and developing molecular markers in orchardgrass (Dactylis Glomerata L.) through transcriptome analysis. Mol. Ecol. Resour. 15 (6), 1497-1509.

Huerta-Cepas, Jaime, Szklarczyk, Damian, Forslund, Kristoffer, Cook, Helen, Heller, Davide, Walter, Mathias C., Rattei, Thomas, Mende, Daniel R., Sunagawa, Shinichi, Kuhn, Michael, 2015. EggNOG 4.5: a hierarchical orthology framework with improved functional annotations for eukaryotic, prokaryotic and viral sequences. Nucleic Acids Res. 44 (D1), D286-D293.

Huerta-Cepas, Jaime, Forslund, Kristoffer, Coelho, Luis Pedro, Szklarczyk, Damian, Jensen, Lars Juhl, von Mering, Christian, Bork, Peer, 2017. Fast genome-wide functional annotation through orthology assignment by EggNOG-mapper. Mol. Biol. Evol. 34 (8), 2115-2122.

Jia, Xin, Sun, Chuangshu, Zuo, Yongchun, Li, Guangyue, Li, Guobin, Ren, Liangyu, Chen, Guilin, 2016. Integrating transcriptomics and metabolomics to characterise the response of Astragalus Membranaceus Bge. Var. mongolicus (Bge.) to progressive drought stress. BMC Genomics 17 (1), 188.

Joshi, Rohit, Wani, Shabir H., Singh, Balwant, Bohra, Abhishek, Dar, Zahoor A., Lone, Ajaz A., Pareek, Ashwani, Singla-Pareek, Sneh L., 2016. Transcription factors and plants response to drought stress: current understanding and future directions. Front. Plant Sci. 7, 1029.

Kanehisa, Minoru, Furumichi, Miho, Mao, Tanabe, Sato, Yoko, Morishima, Kanae, 2016 KEGG: new perspectives on genomes, pathways, diseases and drugs. Nucleic Acids Res. 45 (D1), D353-D361.

Kirtman, B., Power, S.B., Adedoyin, A.J., Boer, G.J., Bojariu, R., Camilloni, I., ... Prather, M., 2013. Near-term climate change: projections and predictability.

Krasensky, Julia, Jonak, Claudia, 2012. Drought, salt, and temperature stress-induced metabolic rearrangements and regulatory networks. J. Exp. Bot. 63 (4), 1593-1608.

Kunstler, Georges, Falster, Daniel, Coomes, David A., Hui, Francis, Kooyman, Robert M. Laughlin, Daniel C., Poorter, Lourens, Vanderwel, Mark, Vieilledent, Ghislain, Wright, S. Joseph, 2016. Plant functional traits have globally consistent effects on competition. Nature 529 (7585), 204.

Langmead, Ben, Salzberg, Steven L., 2012. Fast gapped-read alignment with bowtie 2 Nat. Methods 9 (4), 357.

Lee, Yang Ping, Giorgi, Federico M., Lohse, Marc, Kvederaviciute, Kotryna, Klages, Sven, Usadel, Björn, Meskiene, Irute, Reinhardt, Richard, Hincha, Dirk K., 2013. Transcriptome sequencing and microarray design for functional genomics in the extremophile Arabidopsis relative Thellungiella Salsuginea (Eutrema Salsugineum). BMC Genomics 14 (1), 793.

Lenka, Sangram K., Katiyar, Amit, Chinnusamy, Viswanathan, Bansal, Kailash C., 2011. Comparative analysis of drought-responsive transcriptome in Indica rice genotypes with contrasting drought tolerance. Plant Biotechnol. J. 9 (3), 315-327.

Li, Bo, Dewey, Colin N., 2011. RSEM: accurate transcript quantification from RNA-Sec data with or without a reference genome. BMC Bioinf. 12 (1), 323.

Li, Heng, Handsaker, Bob, Wysoker, Alec, Fennell, Tim, Ruan, Jue, Homer, Nils, Marth, Gabor, Abecasis, Goncalo, Durbin, Richard, 2009. The sequence alignment/map format and SAMtools. Bioinformatics 25 (16), 2078-2079.

Liang, Chunbo, Wang, Wenjun, Wang, Jing, Ma, Jun, Li, Cen, Zhou, Fei, Zhang, Shuquan, Yu, Ying, Zhang, Liguo, Li, Weizhong, 2017. Identification of differentially expressed genes in sunflower (Helianthus Annuus) leaves and roots under drought stress by RNA sequencing. Bot. Stud. 58 (1), 42.

Liu, D., Estiarte, M., Ogaya, R., Yang, X., Peñuelas, J., 2017. Shift in community structure in an early-successional Mediterranean shrubland driven by long-term experimental warming and drought and natural extreme droughts. Glob. Chang. Biol. 23 (10), 4267-4279.

Matsui, Akihiro, Ishida, Junko, Morosawa, Taeko, Mochizuki, Yoshiki, Kaminuma, Eli, Endo, Takaho A., Okamoto, Masanori, Nambara, Eiji, Nakajima, Maiko, Kawashima, Makiko, 2008. Arabidopsis transcriptome analysis under drought, cold, high-salinity and ABA treatment conditions using a tiling array. Plant Cell Physiol. 49 (8), 1135-1149.

Mayaux, Philippe, Bartholomé, Etienne, Fritz, Steffen, Belward, Alan, 2004. A new landcover map of Africa for the year 2000. J. Biogeogr. 31 (6), 861-877.

Moret-Fernández, D., Vicente, J., Latorre, B., Lera, F., Castañeda, C., López, M.V. Herrero, J., 2012. TDR pressure cell for monitoring water content retention and bulk electrical conductivity curves in undisturbed soil samples. Hydrol. Process. 26 (2), $246-254$.

Moriya, Yuki, Itoh, Masumi, Okuda, Shujiro, Yoshizawa, Akiyasu C., Kanehisa, Minoru, 2007. KAAS: an automatic genome annotation and pathway reconstruction server. Nucleic Acids Res. 35 (suppl_2), W182-W185.

Moumeni, Ali, Satoh, Kouji, Kondoh, Hiroaki, Asano, Takayuki, Hosaka, Aeni, Venuprasad, Ramiah, Serraj, Rachid, Kumar, Arvind, Leung, Hei, Kikuchi, Shoshi, 2011. Comparative analysis of root transcriptome profiles of two pairs of drought tolerant and susceptible Rice near-isogenic lines under different drought stress. BMC Plant Biol. 11 (1), 174.

Mun, Bong-Gyu, Hussain, Adil, Park, Eung-Jun, Lee, Sang-Uk, Sharma, Arti, Imran, Qari Muhammad, Jung, Ki-Hong, Yun, Byung-Wook, 2017. Profile and time-scale dynamics of differentially expressed genes in transcriptome of Populus Davidiana under 
drought stress. Plant Mol. Biol. Report. 35 (6), 647-660.

Nelissen, H., Sun, X.H., Rymen, B., Jikumaru, Y., Kojima, M., Takebayashi, Y., ... De Block, J., 2018. The reduction in maize leaf growth under mild drought affects the transition between cell division and cell expansion and cannot be restored by elevated gibberellic acid levels. Plant Biotechnol. J. 16 (2), 615-627.

Osada, Naoki, Hashimoto, Katsuyuki, Kameoka, Yosuke, Hirata, Makoto, Tanuma, Reiko, Uno, Yasuhiro, Inoue, Itsuro, Hida, Munetomo, Suzuki, Yutaka, Sugano, Sumio, 2008. Large-scale analysis of Macaca fascicularis transcripts and inference of genetic divergence between $M$. fascicularis and M. mulatta. BMC Genomics 9 (1), 90.

Pereira, S.S., Guimarães, F.C.M., Carvalho, J.F.C., Stolf-Moreira, R., Oliveira, M.C.N., Rolla, A.A.P., Farias, J.R.B., Neumaier, N., Nepomuceno, A.L., 2011. Transcription factors expressed in soybean roots under drought stress. Genet. Mol. Res. 3689-3701.

Rabara, Roel C., Prateek, Tripathi, Neil Reese, R., Rushton, Deena L., Alexander, Danny, Timko, Michael P., Shen, Qingxi J., Rushton, Paul J., 2015. Tobacco drought stress responses reveal new targets for Solanaceae crop improvement. BMC Genomics 16 (1), 484.

Robinson, Mark D., McCarthy, Davis J., Smyth, Gordon K., 2010. EdgeR: a bioconductor package for differential expression analysis of digital gene expression data. Bioinformatics 26 (1), 139-140.

Shanker, Arun K., Maheswari, M., Yadav, S.K., Desai, S., Bhanu, Divya, Attal, Neha Bajaj, Venkateswarlu, B., 2014. Drought stress responses in crops. Funct. Integr. Genomics 14 (1), 11-22.

Simão, Felipe A., Waterhouse, Robert M., Ioannidis, Panagiotis, Kriventseva, Evgenia V., Zdobnov, Evgeny M., 2015. BUSCO: assessing genome assembly and annotation completeness with single-copy orthologs. Bioinformatics 31 (19), 3210-3212.

Solomon, S., Qin, D., Manning, M., Averyt, K., Marquis, M. (Eds.), 2007. Climate change 2007-the physical science basis: Working group I contribution to the fourth assessment report of the IPCC (Vol. 4). Cambridge university press.

Sprenger, Heike, Kurowsky, Christina, Horn, Renate, Erban, Alexander, Seddig, Sylvia, Rudack, Katharina, Fischer, Axel, Walther, Dirk, Zuther, Ellen, Köhl, Karin, 2016. The drought response of potato reference cultivars with contrasting tolerance. Plant Cell Environ. 39 (11), 2370-2389.

Todaka, Daisuke, Yu, Zhao, Yoshida, Takuya, Kudo, Madoka, Kidokoro, Satoshi, Mizoi, Junya, Kodaira, Ken-Suke, Takebayashi, Yumiko, Kojima, Mikiko, Sakakibara, Hitoshi, 2017. Temporal and spatial changes in gene expression, metabolite accumulation and phytohormone content in rice seedlings grown under drought stress conditions. Plant J. 90 (1), 61-78.

Tricker, Penny J., ElHabti, Abdeljalil, Schmidt, Jessica, Fleury, Delphine, 2018. The physiological and genetic basis of combined drought and heat tolerance in wheat. J. Exp. Bot. ery081.

Tripathi, Abhinandan Mani, Yadav, Amrita, Saikia, Siddhartha Proteem, Roy, Sribash, 2017. Global gene expression pattern in a forest tree species, Tectona Grandis (Linn. F.), under limited water supply. Tree Genet. Genomes 13 (3), 66.

Tyree, Melvin T., Vargas, Gustavo, Engelbrecht, Bettina M.J., Kursar, Thomas A., 2002 Drought until death do us part: a case study of the desiccation-tolerance of a tropical moist forest seedling-tree, Licania Platypus (Hemsl.) Fritsch. J. Exp. Bot. 53 (378), 2239-2247.

Vivien, J.J., Faure, J.J., 1985. Arbres des forêts denses d'Afrique centrale.
Wegrzyn, Jill L., Whalen, Jeanne, Kinlaw, Claire S., Harry, David E., Puryear, Jeffrey, Loopstra, Carol A., Gonzalez-Ibeas, Daniel, Vasquez-Gross, Hans A., Famula, Randi A., Neale, David B., 2016. Transcriptomic profile of leaf tissue from the leguminous tree, Millettia Pinnata. Tree Genet. Genomes 12 (3), 44.

Wong, Melissa M.L., Cannon, Charles H., Wickneswari, Ratnam, 2011. Identification of lignin genes and regulatory sequences involved in secondary Cell Wall formation in Acacia Auriculiformis and Acacia Mangium via de novo transcriptome sequencing. BMC Genomics 12 (1), 342.

Wuebbles, Donald J., Kunkel, Kenneth, Wehner, Michael, Zobel, Zachary, 2014. Severe weather in United States under a changing climate. EOS Trans. Am. Geophys. Union 95 (18), 149-150.

Yang, Zemao, Dai, Zhigang, Lu, Ruike, Wu, Bibo, Tang, Qing, Xu, Ying, Cheng, Chaohua, Jianguang, Su., 2017. Transcriptome analysis of two species of jute in response to polyethylene glycol (PEG)-induced drought stress. Sci. Rep. 7 (1), 16565.

Yang, J., Tian, H., Pan, S., Chen, G., Zhang, B., Dangal, S., 2018. Amazon drought and forest response: Largely reduced forest photosynthesis but slightly increased canopy greenness during the extreme drought of 2015/2016. Glob. Chang. Biol. 24 (5), 1919-1934.

Yates, Steven A., Swain, Martin T., Hegarty, Matthew J., Chernukin, Igor, Lowe, Matthew, Allison, Gordon G., Ruttink, Tom, Abberton, Michael T., Jenkins, Glyn, Skøt, Leif, 2014. De novo assembly of red clover transcriptome based on RNA-Seq data provides insight into drought response, gene discovery and marker identification. BMC Genomics 15 (1), 453.

Ye, Jia, Fang, Lin, Zheng, Hongkun, Zhang, Yong, Chen, Jie, Zhang, Zengjin, Wang, Jing, Li, Shengting, Li, Ruiqiang, Bolund, Lars, 2006. WEGO: a web tool for plotting GO annotations. Nucleic Acids Res. 34 (suppl_2), W293-W297.

Yildırım, Kubilay, Kaya, Zeki, 2017. Gene regulation network behind drought escape, avoidance and tolerance strategies in black poplar (Populus Nigra L.). Plant Physiol. Biochem. 115, 183-199.

Young, Matthew D., Wakefield, Matthew J., Smyth, Gordon K., Oshlack, Alicia, 2010. Gene ontology analysis for RNA-Seq: accounting for selection bias. Genome Biol. 11 (2), R14.

de Zelicourt, Axel, Colcombet, Jean, Hirt, Heribert, 2016. The role of MAPK modules and ABA during abiotic stress signaling. Trends Plant Sci. 21 (8), 677-685.

Zhang, Zhengfeng, Xiao, Benze, 2018. Comparative alternative splicing analysis of two contrasting rice cultivars under drought stress and association of differential splicing genes with drought response QTLs. Euphytica 214 (4), 73.

Zhang, Ji-Yi, Cruz De Carvalho, Maria H., Torres-Jerez, Ivone, Kang, Yun, Allen, Stacy N., Huhman, David V., Tang, Yuhong, Murray, Jeremy, Sumner, Lloyd W., Udvardi, Michael K., 2014. Global reprogramming of transcription and metabolism in Medicago Truncatula during progressive drought and after rewatering. Plant Cell Environ. 37 (11), 2553-2576.

Zheng, Yi, Jiao, Chen, Sun, Honghe, Rosli, Hernan G., Pombo, Marina A., Zhang, Peifen, Banf, Michael, Dai, Xinbin, Martin, Gregory B., Giovannoni, James J., 2016. ITAK: a program for genome-wide prediction and classification of plant transcription factors, transcriptional regulators, and protein kinases. Mol. Plant 9 (12), 1667-1670.

Zhu, Jian-Kang, 2016. Abiotic stress signaling and responses in plants. Cell 167 (2) 313-324. 